TRANSACTIONS OF THE

AMERICAN MATHEMATICAL SOCIETY

Volume 349, Number 4, April 1997, Pages 1333-1358

S 0002-9947(97)01830-8

\title{
DISJOINT PATHS, PLANARIZING CYCLES, AND SPANNING WALKS
}

\author{
XINGXING YU
}

\begin{abstract}
We study the existence of certain disjoint paths in planar graphs and generalize a theorem of Thomassen on planarizing cycles in surfaces. Results are used to prove that every 5-connected triangulation of a surface with sufficiently large representativity is hamiltonian, thus verifying a conjecture of Thomassen. We also obtain results about spanning walks in graphs embedded in a surface with large representativity.
\end{abstract}

\section{INTRODUCTION}

We begin with the definition of a $k$-walk. For a positive integer $k$, a $k$-walk in a graph is a walk that visits every vertex at least once and at most $k$ times. Clearly a 1-walk is just a Hamilton cycle.

It is well known that not all 3-connected planar graphs are hamiltonian (for example, see [1]) and that every triangulation of the sphere with no separating triangles is hamiltonian [17]. Tutte [16] proved that every 4-connected planar graph has a Hamilton cycle. Extending Tutte's technique, Thomassen [12] proved that every 4-connected planar graph is in fact Hamilton connected. Thomassen's theorem implies that every 4-connected planar graph minus an arbitrary vertex is hamiltonian. Plummer [9] conjectured that every 4-connected planar graph minus two arbitrary vertices is hamiltonian. This conjecture was proved by Thomas and $\mathrm{Yu}$ [10] (by extending the techniques of Thomassen in various ways). With some additional techniques, it was also proved in [10] that every edge in a 4-connected projective-planar graph is contained in a Hamilton cycle, which implies the conjecture of Grünbaum [6] that every 4-connected projective-planar graph has a Hamilton cycle.

Grünbaum [6] and Nash-Williams [8] also conjectured that every 4-connected toroidal graph has a Hamilton cycle. While this is still unsolved, Thomassen [12] showed that there are 4-connected toroidal graphs in which certain edges are not contained in any Hamilton cycle. It is shown in [11] that every edge in a 5-connected graph in the torus is contained in a Hamilton cycle. The technique used in [11] can also be used to solve the conjecture of Molluzzo [7] that 6-connected toroidal graphs are Hamilton connected. We also mention that Duke [3] gave a function $c(g)$ for 2-connected graphs with the property that such a graph with orientable genus $g$

Received by the editors August 20, 1993.

1991 Mathematics Subject Classification. Primary 05C38, 05C10, 57M15.

Key words and phrases. bridge, disjoint paths, embedding, Hamilton cycle, representativity, walk.

Partially supported by NSF grants DMS-9105173 and DMS-9301909.

(C)1997 American Mathematical Society 
and connectivity at least $c(g)$ is hamiltonian. In general, this function is not best possible. For $g=0$ the best value is 4 by Tutte [16]. For $g=1$ the above-mentioned conjecture of Grünbaum and Nash-Williams would imply that the correct value is 4.

It is not difficult to see that the existence of a $k$-walk in a graph implies the existence of a spanning tree with maximum degree $k+1$. Thomassen [14] showed that every triangulation of an orientable surface of genus $g$ with no noncontractible cycle of length less than $2^{3 g+4}$ contains a spanning tree of maximum degree at most 4. This upper bound 4 is best possible. He also raised two related conjectures in [14]. The first states that every 4-connected triangulation of an orientable surface with sufficiently large representativity has a spanning tree with maximum degree at most three. This was established by Ellingham and Gao [4]. The second conjecture in [14] states that every 5 -connected triangulation of an orientable surface with sufficiently large representativity is hamiltonian. In this paper, we prove this conjecture for both orientable and non-orientable surfaces. We also prove that 4-connected graphs embedded in surfaces with large representativity admits 2-walks, which generalizes and improves a result of Ellingham and Gao [4]. We finally answer a question of Ellingham (personal communication) about the existence of a 3-walk in a 3connected graph, which improves a result of Thomassen [14] on spanning trees. The large representativity condition in all the above results is essential as Archdeacon, Hartsfield and Little (reported in [4]) have shown the following: for each $n$ there is a triangulation of some surface which is $n$-connected and has representativity at least $n$, and in which every spanning tree has maximum degree at least $n$.

In order to state our results precisely, we need some terminology. We follow that of Bondy and Murty [1]. Only simple graphs will be considered here. Let $G$ and $H$ be two graphs. The intersection and the union of $G$ and $H$, denoted by $G \cap H$ and $G \cup H$, are the graphs $(V(G) \cap V(H), E(G) \cap E(H))$ and $(V(G) \cup V(H), E(G) \cup$ $E(H)$ ), respectively. A block of a graph $G$ is a maximal 2-connected subgraph of $G$. We view $K_{2}$ as being 2 -connected. For any path $P$ and $x, y \in V(P) \cup E(P)$, we use $x P y$ to denote the subpath of $P$ between $x$ and $y$, where $x P y$ does not include $x$ if $x \in E(P)$ or $y$ if $y \in E(P)$

Let $P$ be a subgraph of a graph $G$. Let $S$ be a component of $G-V(P)$, and let $B$ be the union of $S$ and all edges with one end in $V(S)$ and the other in $V(P)$. Then $B$ is called a $P$-bridge of $G$. Every vertex in $a(B)=V(B) \cap V(P)$ is said to be an attachment of $B$ on $P$. This notation should not cause any confusion (as we will always say that "for a $P$-bridge $B$ of $G$ "). In the sequel $P$ will always be a path or a set of disjoint paths.

A plane graph is a graph embedded in the plane so that no two edges cross. A plane subgraph $H$ of a plane graph $G$ is a subgraph of $G$ inheriting the embedding of $G$. Given a connected plane graph, the boundary of the infinite face is called the outerwalk of the graph, or outercycle if the graph is 2-connected. A walk is a facial walk in a plane graph if it bounds a face of the graph. Suppose that $C$ is a facial walk of a plane graph $G$ and that $x_{1}, \cdots, x_{m}$ are distinct vertices or edges of $G$ on $C$. Then we say that $x_{1}, \cdots, x_{m}$ appear on $C$ in this order if walking along $C$ in the clockwise order from $x_{1}$ to $x_{m}$ we encounter $x_{i}$ before $x_{i+1}$ and after the first visit to $x_{i+1}$ we never encounter $x_{1}, \cdots, x_{i}$ again. Given $x, y \in E(C) \cup V(C)$ on a facial walk $C$ in a plane graph, we use $x C y$ to denote the minimal subwalk of $C$ from $x$ to $y$ in the clockwise order, where $x C y$ does not include $x$ if $x \in E(C)$ or $y$ if $y \in E(C)$. 
For convenience, we adopt the following convention. Let $G$ be a plane graph with outerwalk $C$, and let $x$ and $y$ be two vertices on $C$. Then $G+x y$ (or $G+y x$ ) denotes the plane graph obtained from $G$ by adding an edge between $x$ and $y$ so that $(C-(x C y-\{x, y\}))+x y$ (or $(C-(y C x-\{x, y\}))+y x)$ is the outerwalk of the resulting graph.

Finally, we need some parameters for graphs embedded in surfaces. We refer to [13] for other related concepts. Suppose that $G$ is embedded in a surface $\Sigma$. We usually do not distinguish between $G$ and its embedding in $\Sigma$. A cycle of $G$ is noncontractible if it is homotopically nontrivial in $\Sigma$, otherwise it is contractible. The representativity (or face-width) of $G$, denoted by $\rho(G, \Sigma)$, is $\min \{|\Gamma \cap V(G)|: \Gamma$ is a homotopically nontrivial simple closed curve in $\Sigma$ and $\Gamma \cap G \subset V(G)\}$. (The edge-width of $G$ is the length of a shortest noncontractible cycle of $G$, which is the same as representativity when the graph is a triangulation.) We use $d_{G \Sigma}(x, y)$ to denote $\min \{|\Gamma \cap V(G)|: \Gamma$ is a simple curve in $\Sigma$ from $x$ to $y$ and $\Gamma \cap G \subset V(G)\}$. For two disjoint vertex sets $C$ and $D$ in $G$, let $d_{G \Sigma}(C, D)=\min \left\{d_{G \Sigma}(x, y): x \in C\right.$ and $y \in D\}$.

We now outline the rest of the paper. In Section 2 we prove a modification lemma which will be used in the proofs in Section 3 on disjoint paths. In Section 4 we generalize Thomassen's results on planarizing cycles of triangulations to general graphs. In Section 5 we prove Thomassen's conjecture that every 5-connected triangulation with large representativity has a Hamilton cycle. In Section 6 we find 2-walks in 4-connected graphs with large representativity and 3-walks in 3connected graphs with large representativity.

\section{Preliminary Results}

Throughout the rest of the paper, when we say "by Theorem $n$ (or by Lemma $n$ ), we find a path $P$ " we mean that the path $P$ must satisfy the relevant conclusions. Before we are able to describe a type of modification which is needed to simplify our statements, we need several lemmas. The first result (see [11], also independently obtained by D. P. Sanders) ensures a path between two specified vertices and using a specified edge.

Lemma 2.1. Let $G$ be a connected plane graph with outerwalk $C$ and let $u, v \in$ $V(G)$ and $e \in E(C)$. Suppose that $G$ has a path from $u$ to $v$ using the edge e. Then there is a path $P$ in $G$ from $u$ to $v$ using e such that for every $P$-bridge $B$ of $G$, (i) $|a(B)| \leq 3$, and (ii) $|a(B)| \leq 2$ if $(B-a(B)) \cap C \neq \emptyset$.

The following result [10] guarantees a path between two given vertices which does not use two other specified vertices.

Lemma 2.2. Let $G$ be a connected plane graph with outerwalk $C$, and let $x, y, u, v$ be four distinct vertices on $C$ in this order such that $u, v \notin x C y$. Then $G$ contains a path $P$ from $x$ to $y$ missing $u$ and $v$ such that for every $(P \cup\{u, v\})$-bridge $B$ of $G$, (i) $|a(B)| \leq 3$, and (ii) $|a(B)| \leq 2$ if $(B-a(B)) \cap x C y \neq \emptyset$.

The next lemma is similar to Lemma 2.2, and ensures a path between two given vertices and not using three specified vertices. The proof of Lemma 2.3 is similar to that of Lemma 2.7 .

Lemma 2.3. Let $G$ be a connected plane graph with outerwalk $C$, and let $x, y, u, v, w$ be five distinct vertices on $C$ in this order such that $u, v, w \notin x C y$. Then $G$ has a 
path $P$ from $x$ to $y$ missing $u, v, w$ such that for every $(P \cup\{u, v, w\})$-bridge $B$ of $G$, (i) $|a(B)| \leq 4$, (ii) $|a(B)| \leq 2$ if $(B-a(B)) \cap x C y \neq \emptyset$.

The following result [10] finds a path between two vertices using two specified edges.

Lemma 2.4. Let $G$ be a connected plane graph with outerwalk $C$, and let $u, v \in$ $V(C)$ and $e, f \in E(C)$ so that $u, e, f, v$ appear on $C$ in this order and $G$ has a path from $u$ to $v$ using $e$ and $f$. Then $G$ has a path $P$ from $u$ to $v$ using $e$ and $f$ such that for every $P$-bridge $B$ of $G$, (i) $|a(B)| \leq 3$, and (ii) $|a(B)| \leq 2$ if $(B-a(B)) \cap u C v \neq \emptyset$.

For later convenience, we need another simple lemma.

Lemma 2.5. Let $G$ be a connected plane graph with outerwalk $C$, and let $x, y, z \in$ $V(C)$ in this order such that $G-z$ has a path from $x$ to $y$. Then $G$ has a path $P$ from $x$ to $y$ not using $z$ such that for every $(P \cup\{z\})$-bridge $B$ of $G$, (i) $|a(B)| \leq 3$, and (ii) $|a(B)| \leq 2$ if $(B-a(B)) \cap x C z \neq \emptyset$.

Proof. We consider $G+z x$. By Lemma 2.1 we find a path $Q$ in the new graph from $y$ to $z$ using $z x$. Then $P=Q-z x$ is the desired path.

We are now ready to state our modification lemma, which will be used heavily in proofs in next section. The conditions in this lemma may seem complicated, but they are easy to verify when applied.

Lemma 2.6 (The Modification Lemma). Let $G$ be a connected plane graph with outerwalk $C$, and let $\alpha$ and $\beta$ be two vertices on $C$ with $L=\alpha C \beta$ simple. Let $H$ be a plane subgraph of $G-V(L)$ with outerwalk $D$ such that each $(H \cup L)$-bridge of $G$ has at least one attachment on $L$ and at most one attachment on $H$. Suppose there are distinct vertices $u$ and $w$ in $D \cap C$ such that $u D w$ is simple, $\alpha, \beta, w$, $u$ are on $C$ in this order, and all attachments of $(H \cup L)$-bridges on $H$ are contained in $u D w$. Suppose that $Q$ is a subgraph of $H$ containing $u$ and $w$ such that for every $Q$-bridge $B$ of $H$, (1) $|a(B)| \leq 4$, (2) $|a(B)| \leq 3$ if $(B-a(B)) \cap u D w \neq \emptyset$, (3) $B \cap u D w$ is a simple path.

Then there is a path $P$ in $G-V(Q)$ from $\alpha$ to $\beta$ such that for every $(P \cup Q)$-bridge $B$ of $G$, (i) $|a(B)| \leq 4$ and (ii) $|a(B)| \leq 2$ if $(B-a(B)) \cap V(L) \neq \emptyset$.

Note that in Lemma 2.6 we may allow $u=w$ and let $u D w=D$. This note is used in the proof of Lemma 3.3.

Proof of Lemma 2.6. Since $G$ is a plane graph, we can divide $L$ into non-trivial edge-disjoint subpaths $x L y$ of three types according to the $Q$-bridges of $H$ : (a) for some $Q$-bridge $B$ of $H, x L y$ is a maximal subpath of $L$ such that $x$ and $y$ belong to some $(H \cup L)$-bridges of $G$ each with an attachment in $B-a(B)$; (b) for some vertex $v$ of $Q, x L y$ is the maximal subpath of $L$ such that both $x$ and $y$ belong to some $(H \cup L)$-bridges of $G$ with $v$ as an attachment; (c) the remaining non-trivial edge maximal subpaths of $L$.

Obviously, each edge of $L$ belongs to exactly one of these subpaths. We label these subpaths (from $\alpha$ to $\beta$ ) as $L_{i}, i=1, \cdots, m$. Let the endvertices of $L_{i}$ be $x_{i}$ and $x_{i+1}$ such that $x_{1}, \cdots, x_{m}$ are on $C$ in this order, where $x_{1}=\alpha$ and $x_{m+1}=\beta$. By (3), distinct $L_{i}$ 's of type (a) are associated with distinct $Q$-bridges of $H$. 
If $L_{i}$ is of type (a), then in the union of $L_{i}, B$, and all $(H \cup L)$-bridges of $G$ with all their attachments in $L_{i} \cup(B-a(B))$, we find a path $P_{i}$ from $x_{i}$ to $x_{i+1}$ not using vertices of $a(B)$ (Lemma 2.2 or 2.3 ).

If $L_{i}$ is of type (b), then in the union of $L_{i}$ and all $(H \cup L)$-bridges of $G$ with attachments in $L_{i} \cup\{v\}$, we find a path $P_{i}$ from $x_{i}$ to $x_{i+1}$ not using $v$ (Lemma $2.5)$.

If $L_{i}$ is of type (c), then in the union of $L_{i}$ and all $(H \cup L)$-bridges of $G$ whose attachments are in $L_{i}$, we find a path $P_{i}$ from $x_{i}$ to $x_{i+1}$ (Lemma 2.1).

It is now easy to see that $P=\bigcup_{i=1}^{m} P_{i}$ is the desired path.

Remark. If in Lemma 2.6 we ask that for every $Q$-bridge $B$ of $H$ we have that $|a(B)| \leq 3$ and that $|a(B)| \leq 2$ if $(B-a(B)) \cap u D w \neq \emptyset$, then we can require that $|a(B)| \leq 3$ for every $(P \cup Q)$-bridge $B$ of $G$. The proof is exactly the same as that for Lemma 2.6 except in modifying $L_{i}$ 's of type (a), we only apply Lemma 2.2. This remark is used in the proof of Lemma 3.3.

We note that the modification of $L$ (to get $P$ ) takes place in the union of $L=$ $\alpha C \beta, H$ and all $(H \cup L)$-bridges of $G$ with attachments in $H \cup L$. In the rest of the paper, when we apply Lemma 2.6 we may simply say by modifying $\alpha C \beta$ with respect to $(H, Q)$ we get a path $P$. We finish this section with a result slightly stronger than Lemma 2.6. But first we need the following easy lemma.

Lemma 2.7. Let $G$ be a connected plane graph with outerwalk $C$, and let $x, y, u, v, w$ be five distinct vertices on $C$ in this order with $u, v, w \notin x C y$. Then $G$ contains a path $P$ from $x$ to $y$ missing $u, v$ and $w$ such that for every $(P \cup\{u, v, w\})$-bridge $B$ of $G$, (i) $|a(B)| \leq 4$, (ii) $|a(B)| \leq 3$ if $(B-a(B)) \cap w C u \neq \emptyset$, and (iii) if $(B-a(B)) \cap w C u \neq \emptyset$ then $(B-a(B)) \cap u C w=\emptyset$.

Proof. Let $H$ be the union of $x C y$ and all blocks of $G-\{u, v, w\}$ containing an edge of $x C y$. Let $D$ be the outerwalk of $H$. Let $s, t \in y D x$ (in this order on $D$ ) with $s D t$ shortest such that $s$ and $t$ are the attachments of some $(H \cup\{u, v, w\})$-bridges of $G$ containing $u$ and $w$ respectively. In $H$ we use Lemma 2.4 to find a path $P$ from $x$ to $y$ using $s$ and $t$ ( $s=t$ is possible). It is easy to verify that $P$ is the desired path. In fact there is at most one $P$-bridge of $G$ with 4 attachments on $P$ (which occurs only if $s=t$ ).

Lemma 2.8. Let $G, L, C, H, D, u, w, Q$ be as in Lemma 2.6, and let $w_{1}, \cdots, w_{m} \in$ $D$ (in this order on $D)$ be the attachments of $(H \cup L)$-bridges of $G$. Let $s_{i} L t_{i}$ be the maximal subpath of $L$ such that $s_{i}$ and $t_{i}$ belong to some $(H \cup L)$-bridges of $G$ with $w_{i}$ as an attachment and $s_{i} C t_{i}=s_{i} L t_{i}$, and let $\alpha, s_{1}, \cdots, s_{m}, \beta$ appear on $C$ in this order. Suppose $z_{1}$ and $z_{2}$ are vertices on $L\left(z_{1}=z_{2}\right.$ is possible) such that none of the following occurs: (I) $z_{1}, z_{2} \in s_{i} L t_{i}-\left\{s_{i}, t_{i}\right\}$ for some $i$, or (II) $z_{1}, z_{2} \in t_{i} L s_{i+1}-\left\{t_{i}, s_{i+1}\right\}$ for some $i$.

Then we can find a path $P$ as in Lemma 2.6 from $\alpha$ to $\beta$ with an extra condition: either $\left\{z_{1}, z_{2}\right\} \cap P \neq \emptyset$ or $z_{1}$ and $z_{2}$ belong to distinct $(P \cup Q)$-bridges of $G$.

Proof. As in the proof of Lemma 2.6, we divide $L$ into edge-disjoint paths $L_{i}=$ $x_{i} L x_{i+1}$ of three types. It is clear that if $z_{1}\left(\right.$ or $\left.z_{2}\right)$ does not belong to any $L_{i}-$ $\left\{x_{i}, x_{i+1}\right\}$ which is of type (a) or (b), then this path $P$ can easily be found to use $z_{1}$ (or $z_{2}$ ). It is also clear that we may assume that $z_{1}, z_{2} \in L_{i}-\left\{x_{i}, x_{i+1}\right\}$ for some $i$. Since (I) does not occur, we may assume that $L_{i}$ is of type (a). Let $B$ be the $Q$-bridge of $H$ associated with $L_{i}$, and let $w_{a}, \cdots, w_{b} \in B-a(B)$ in this order on 
$D$ (where $s_{a}=x_{i}$ and $t_{b}=x_{i+1}$ ). We now show how to modify $L_{i}$ to get a path $P_{i}$ to form the desired path $P$. We use $J_{j}$ to denote the union of $s_{j} L t_{j}$ and all $(H \cup L)$-bridges of $G$ with all attachments contained in $s_{j} L t_{j} \cup\left\{w_{j}\right\}$.

Suppose first that $z_{1} \in s_{k} L t_{k}-\left\{s_{k}, t_{k}\right\}$. Again since (I) does not occur, $z_{2} \notin$ $s_{k} L t_{k}-\left\{s_{k}, t_{k}\right\}$. Since $z_{1}, z_{2} \in L_{i}-\left\{x_{i}, x_{i+1}\right\}$, by symmetry we may assume that $w_{k+1} \in B-a(B)$ (the other case that $w_{k-1} \in B-a(B)$ can be treated similarly). Then we find a path $R$ in $B$ from $w_{k}$ to $w_{k+1}$ not using vertices of $a(B)$ (Lemma 2.2 or Lemma 2.7). In $J_{k}$ we find a path $R_{k}$ from $w_{k}$ to $s_{k}$ using $z_{1}$ and $t_{k}$ (Lemma 2.4 ) if $t_{k} \neq s_{k+1}$; otherwise we simply find $R_{k}$ from $w_{k}$ to $s_{k}$ not using $t_{k}$ (Lemma 2.5). In $J_{k+1}$ we find a path $R_{k+1}$ from $w_{k+1}$ to $t_{k+1}$ using $s_{k+1}$ (Lemma 2.1). We then use Lemma 2.6 to modify $x_{i} L_{i} s_{k}$ and $t_{k+1} L_{i} x_{i+1}$ (both with respect to $(B, R \cup a(B)))$ to complete $P_{i}$ (together with $R, R_{k}, R_{k+1}$ ).

Hence we may assume that $z_{1} \in t_{k} L s_{k+1}$ with $k$ maximum and $z_{2} \in t_{l} L s_{l+1}$ with $l$ maximum, where $k \leq l$. Thus $t_{k+1} \neq z_{1}$ and $t_{l+1} \neq z_{2}$. Note that if $k=l$, then since (II) does not occur, $\left\{z_{1}, z_{2}\right\} \cap\left\{t_{k}, s_{k+1}\right\} \neq \emptyset$, and in this case we may assume by symmetry that $z_{2}=s_{k+1}$. Thus we may assume that $z_{2} \in s_{k+1} L t_{l+1}$. Since $\left\{z_{1}, z_{2}\right\} \cap\left\{x_{i}, x_{i+1}\right\}=\emptyset$, we can take $c<k$ to be maximum such that $s_{c} \neq z_{1}$ (note that $c=k$ is possible). In $B$ we find a path $R$ from $w_{c}$ to $w_{k+1}$ not using the vertices in $a(B)$ (Lemma 2.2 or Lemma 2.7). In $J_{c}$ we find a path $R_{c}$ from $w_{c}$ to $s_{c}$ using $t_{c}$ if $t_{c} \neq s_{k+1}$ (Lemma 2.1); otherwise $t_{c}=s_{k+1}=z_{1}$ and we just find a path $R_{c}$ in $J_{c}$ from $w_{c}$ to $s_{c}$ not using $t_{c}$ (Lemma 2.5). In $J_{k+1}$ we find a path $R_{k+1}$ from $w_{k+1}$ to $t_{k+1}$ using $s_{k+1}$ (Lemma 2.1). We then modify $x_{i} L_{i} s_{c}$ and $t_{k+1} L_{i} x_{i+1}$ (both with respect to $\left.(B, R \cup a(B))\right)$ to complete $P_{i}$ (together with $R, R_{c}$ and $\left.R_{k+1}\right)$. It is easy to check that $P_{i}$ is as desired.

We remark here that Lemma 2.8 will be used in the proof of Claim 2 in Theorem 3.4 .

\section{DisJOINT PATHS IN PLANAR GRAPHS}

In this section we study the existence of certain disjoint paths in a planar graph. First we deal with disjoint paths among vertices lying on a single facial cycle.

Lemma 3.1. Let $G$ be a 2-connected plane graph with outercycle $C$, let $x, u, v, y$ be four distinct vertices on $C$ in this order, and let e be an edge on yCx. Suppose that $G$ does not have a 2-cut $\{p, q\}$ with $p \in y C e$ and $q \in x C u-\{x\}$ such that the $\{p, q\}$-bridge of $G$ containing $e$ is 2-connected. Then $G$ has two disjoint paths $P$ and $Q$ with $P$ from $x$ to $y$ using $e$ and $Q$ from $u$ to $v$ such that for every $(P \cup Q)$-bridge $B$ of $G$, (i) $|a(B)| \leq 4$, and (ii) $|a(B)| \leq 2$ if $(B-a(B)) \cap y C v \neq \emptyset$.

Proof. Let $H$ be the union of $u C v$ and all blocks of $G-y C x$ containing an edge of $u C v$. Let $w \in V(H) \cap x C u$ with $x C w$ shortest. By Lemma 2.1 we find a path $Q$ in $H$ from $u$ to $v$ using $w$. Let $w_{i} \in H, i=1, \cdots, m$, be the attachments of $(H \cup y C x)$-bridges of $G$ (in this order on the outerwalk of $H$, where $w_{m}=$ $w)$. Let $s_{i} C t_{i}$ be the maximal subpath of $y C x$ such that $s_{i}$ and $t_{i}$ are in some $(H \cup y C x)$-bridges of $G$ with $w_{i}$ as an attachment. Define $J_{i}$ to be the union of $s_{i} C t_{i}$ and all $(H \cup y C x)$ - bridges of $G$ with all attachments contained in $s_{i} C t_{i} \cup\left\{w_{i}\right\}$. We divide $y C x$ into subpaths $L_{j}=x_{j} C x_{j+1}, j=1, \cdots, n$, of types (a), (b) and (c) as in the proof of Lemma 2.6.

If $e \in L_{j}$ of type (c), then in the union of $x_{j} C x_{j+1}$ and all $(H \cup y C x)$-bridges of $G$ with all attachments in $x_{j} C x_{j+1}$ we use Lemma 2.1 to find a path $P_{j}$ from $x_{j}$ to 
$x_{j+1}$ using $e$. We now modify $y C x_{j}$ and $x_{j+1} C x$ (both with respect to $(H, Q)$ ) to get two paths which, together with $P_{j}$, give the desired path $P$ from $x$ to $y$ using $e$. Hence we may assume that $e \in L_{j}$ of type (a) or (b).

Case 1. Suppose that $e \in t_{k} C s_{k+1} \subset L_{j}$ of type (a). Let $B$ be the $Q$-bridge of $H$ associated with $L_{j}$. Then $w_{k}, w_{k+1} \in B-a(B)$. In the union of $t_{k} C s_{k+1}$ and all $(H \cup y C x)$-bridges of $G$ with all attachments contained in $t_{k} C s_{k+1}$, we find a path $S$ from $t_{k}$ to $s_{k+1}$ using $e$ (Lemma 2.1).

(1.1) If there is a $w_{l} \in B-a(B)$ with $l<k$ such that $s_{l} \neq t_{k}$, then take such $w_{l}$ with $l$ maximum. In $B$ we find a path $R$ from $w_{l}$ to $w_{k}$ (Lemma 2.2) not using vertices in $a(B)$. In $J_{k}$ we find a path $R_{k}$ from $w_{k}$ to $t_{k}$ using $s_{k}$ if $s_{k} \neq s_{l}$ (Lemma 2.1 ), otherwise not using $s_{k}$ (Lemma 2.5). In $J_{l}$ we find a path $R_{l}$ from $w_{l}$ to $s_{l}$ using $t_{l}$ if $t_{l} \notin R_{k}$ (Lemma 2.1), otherwise not using $t_{l}$ (Lemma 2.5). Finally modify $y C s_{l}$ and $s_{k+1} C x$ (both with respect to $(H, Q \cup R)$ ) to get two paths which, together with $R, R_{k}, R_{l}$ and $S$, give the desired path $P$.

(1.2) Therefore we may assume that if $w_{l} \in B-a(B)$ with $l<k$ then $s_{l}=t_{k}$. By symmetry we may also assume that if $w_{l} \in B-a(B)$ with $l>k+1$ then $t_{l}=s_{k+1}$. Let $R_{k}=\left\{s_{k}\right\}$ if $s_{k}=t_{k}$; otherwise we find a path $R_{k}$ in $J_{k}$ from $s_{k}$ to $t_{k}$ such that $w_{k} \in R_{k}$ or the $R_{k}$-bridge of $J_{k}$ containing $w_{k}$ has one attachment. We define $R_{k+1}$ similarly. We modify $y C s_{k}$ and $t_{k+1} C x$ (both with respect to $(H, Q)$ ) to get two paths which, together with $S, R_{k}, R_{k+1}$, give the desired path $P$.

Case 2. Hence let $e \in J_{k}$ for some $k$. By our 2-cut assumption, $w_{k} \neq w_{m}$. If $w_{k} \in Q$, then we find a path $P_{k}$ in $J_{k}-w_{k}$ from $s_{k}$ to $t_{k}$ using $e$ (Lemma 2.1). If $w_{k} \in B-a(B)$ for some $Q$-bridge of $H$ and $w_{i} \notin B-a(B)$ for every $i \neq k$, then we find a path $P_{k}$ in $J_{k}$ from $s_{k}$ to $t_{k}$ using $e$ (Lemma 2.1). In either case, we modify $y C s_{k}$ and $t_{k} C x$ (both with respect to $(H, Q)$ ) to obtain two paths which, together with $P_{k}$, give the desired path $P$. Thus let $w_{k} \in B-a(B)$ for some $Q$-bridge $B$ of $H$, and by symmetry we may assume that $w_{k-1} \in B-a(B)$.

(2.1) If there is some $l<k$ such that $w_{l} \in B-a(B)$ and $s_{l} \neq s_{k}$, we select $l$ to be maximum. First we find a path $R$ in $B$ from $w_{l}$ to $w_{k}$ not using vertices in $a(B)$ (Lemma 2.2). Then in $J_{k}$ we find a path $R_{k}$ from $w_{k}$ to $t_{k}$ using $s_{k}$ and $e$ (Lemma 2.4). Finally we find a path $R_{l}$ in $J_{l}$ from $w_{l}$ to $s_{l}$ using $t_{l}$ if $t_{l} \neq s_{k}$ (Lemma 2.1), otherwise not using $t_{l}$ (Lemma 2.5). Now we just modify $y C s_{l}$ and $t_{k} C x$ (both with respect to $(H, Q \cup R))$ to get two paths $P_{1}$ and $P_{2}$. It is easy to check that $P=R \cup R_{k} \cup R_{l} \cup P_{1} \cup P_{2}$ is the desired path.

Hence we may assume that $s_{i}=s_{k}$ for every $w_{i} \in B-a(B)$ with $i<k$ and by symmetry that $t_{i}=t_{k}$ for every $w_{i} \in B-a(B)$ with $i>k$.

(2.2) Suppose $w_{k+1} \notin B-a(B)$. Then $w_{k-1} \in B-a(B)$. First we consider the case that $s_{k}$ is not incident to $e$. If there is a path in $J_{k}-s_{k}$ from $w_{k}$ to $t_{k}$ using $e$, then we let $N$ be the union of such a path and all blocks of $J_{k}-s_{k}$ containing an edge of that path. Let $z \in V(N) \cap C$ with $s_{k} C z$ shortest. By Lemma 2.4 we find a path $R_{k}$ in $J_{k}-s_{k}$ from $w_{k}$ to $t_{k}$ using $z$ and $e$. Now in $B$ we find a path $R$ by Lemma 2.2 from $w_{k}$ to $w_{k-1}$ not using vertices in $a(B)$. In $J_{k-1}$ we find a path $R_{k-1}$ from $w_{k-1}$ to $s_{k-1}=s_{k}$. We modify $y C s_{k}$ and $t_{k} C x$ (both with respect to $(H, Q \cup R))$ to get the desired path $P$. Hence we may assume that such a path in $J_{k}-s_{k}$ from $w_{k}$ to $t_{k}$ using $e$ does not exist, and therefore $G$ has a 2-cut $\left\{s_{k}, p\right\}$ with $p \in e C t_{k}$. We may select this 2 -cut so that $p C t_{k}$ is shortest. 
If $p=t_{k}$, we then just find a path in $J_{k}$ from $s_{k}$ to $t_{k}$ using $e$, and then modify $y C s_{k}$ and $t_{k} C x$ (both with respect to $\left.(H, Q)\right)$ to get the desired path $P$. Hence let $p \neq t_{k}$. Let $T$ be the $\left\{s_{k}, p\right\}$-bridge of $J_{k}$ containing $w_{k}$. Then in $T-s_{k}$ we find a path $R_{1}$ from $p$ to $t_{k}$ such that $w_{k} \in R_{1}$ or the $R_{1}$-bridge of $T-s_{k}$ containing $w_{k}$ has exactly one attachment (Lemma 2.1), and in the other $\left\{s_{k}, p\right\}$-bridge of $J_{k}$ containing $e$, we find a path $R_{2}$ from $s_{k}$ to $p$ using $e$ (Lemma 2.1). Now we can modify $y C s_{k}$ and $t_{k} C x$ (both with respect to $(H, K)$ ) to get the desired path $P$.

This reduces (2.2) to the case that $s_{k}$ is incident to $e$. If $t_{k}$ is also incident to $e$, we then just modify $y C s_{k}$ and $t_{k} C x$ (both with respect to $(H, Q)$ ), and then add the edge $e=s_{k} t_{k}$ to get the desired path $P$. So $t_{k}$ is not incident to $e$. Let $s$ be the end of $e$ other than $s_{k}$ and let $S$ be the union of $s C t_{k}$ and all blocks of $J_{k}-s_{k}$ containing an edge of $s C t_{k}$. Let $p \in S$ such that $p=w_{k}$ if $w_{k} \in S$; otherwise $p \in S$ is the cutvertex of $J_{k}-s_{k}$ separating $w_{k}$ from $s C t_{k}$. Now we find a path in $S$ from $s$ to $t_{k}$ using $p$ (Lemma 2.1), and then we modify $y C s_{k}$ and $t_{k} C x$ (both with respect to $(H, Q))$ to get the desired path $P$ (using $e$ ).

(2.3) Thus by symmetry we may assume that $w_{k-1}, w_{k+1} \in B-a(B)$. If $s_{k} C t_{k}=$ $s_{k} t_{k}$, then we just modify $y C s_{k}$ and $t_{k} C x$ (both with respect to $(H, Q)$ ) to get two paths which, together with $e$, give the desired path $P$. Hence by symmetry we may assume that $s_{k}$ is not incident to $e$.

If in $J_{k}-s_{k}$ there is a path from $w_{k}$ to $t_{k}$ using $e$, we then let $N$ and $z$ be defined as in (2.2), and the same argument in (2.2) also works here. Hence we may assume that such a path does not exist. Again, as in (2.2) we have the same 2-cut $\left\{s_{k}, p\right\}$ with $p C t_{k}$ shortest, and the case for $p=t_{k}$ is the same as in (2.2). So let $p \neq t_{k}$. We first find a path $R$ in $B$ from $w_{k}$ to $w_{k+1}$ not using the vertices in $a(B)$ (Lemma 2.2 ). Let $S$ and $T$ be the $\left\{s_{k}, p\right\}$-bridges of $J_{k}$ containing $e$ and $w_{k}$, respectively. Since $p \neq t_{k}$, in $T-s_{k}$ we can find a path $R_{1}$ from $p$ to $w_{k}$ not using $t_{k}$ (Lemma 2.5). In $S$ we find a path $R_{2}$ from $s_{k}$ to $p$ using $e$ (Lemma 2.1). In $J_{k+1}$ we find a path $R_{3}$ from $w_{k+1}$ to $t_{k+1}=t_{k}$. Finally, we modify $y C s_{k}$ and $t_{k} C x$ (with respect to $(H, Q \cup R))$ to get the desired path $P$ (together with $R, R_{1}, R_{2}$, and $R_{3}$ ).

Lemma 3.2. Let $G$ be a 2-connected plane graph with outercycle $C$, and let $x, y, u$, $v, w$ be five distinct vertices on $C$ in this order. Suppose that $G$ does not have two disjoint paths $X$ and $Y$, where $X$ is from $x$ to $v$ using $w$ and $Y$ is from $y$ to $u$ or $X$ is from $x$ to $w$ and $Y$ is from $y$ to $v$ using $u$, such that for every $(X \cup Y)$-bridge $B$ of $G$, (i) $|a(B)| \leq 4$, and (ii) $|a(B)| \leq 2$ if $(B-a(B)) \cap w C u \neq \emptyset$. Then $G$ has two disjoint paths $V$ and $W$ with $V$ from $x$ to $w$ and $W$ from $y$ to $u$ using $v$ such that for every $(V \cup W)$-bridge $B$ of $G$, (i) $|a(B)| \leq 4$, and (ii) $|a(B)| \leq 2$ if $(B-a(B)) \cap w C u \neq \emptyset$.

Proof. By Lemma 3.1 we know that $G$ has 2-cuts $\{p, q\}$ and $\{r, s\}$, where $v, p, w, x$, $q, y$ and $x, r, y, u, s, v$ appear on $C$ in this order, such that the $\{p, q\}$-bridge $L$ containing $w C x$ and the $\{r, s\}$-bridge $R$ containing $y C u$ are 2-connected. Therefore $x, q, r, y$ must appear on $C$ in this order, which implies that $r \neq x$ and $q \neq y$. (Note that $q=r$ is possible.) Also by Lemma 3.1 we may assume that $p \neq w$ and $s \neq u$ (as we can choose the edge $e$ at $w$ on $v C w$ when applying Lemma 3.1). Let $M=G-((L \cup R)-\{p, q, r, s\})$.

In $L+p w$ we find a path $R_{1}$ from $x$ to $q$ using $p w$ (Lemma 2.1). In $M+p q$ we find a path $R_{2}$ from $r$ to $s$ using $p q$ and $v$ (Lemma 2.4). In $R+s r$ we find a path $R_{3}$ from $y$ to $u$ using $s r$ (Lemma 2.1). Then $\left(R_{1}-p w\right) \cup\left(R_{2}-p q\right) \cup\left(R_{3}-s r\right)$ gives 
the desired paths $V$ and $W$, where $W$ uses $v$. (By a symmetric argument we can ask that $V$ use $v$.)

We now turn our attention to disjoint paths among vertices on two facial cycles.

Lemma 3.3. Let $G$ be a 2-connected plane graph with outercycle $C$ and another facial cycle $D$ disjoint from $C$, and let $x, y \in C$ and $u, v \in D$ be four distinct vertices of $G$. Then $G$ has two disjoint paths $P$ and $Q$ with $P$ from $x$ to $y$ and $Q$ from $u$ to $v$ such that for every $(P \cup Q)$-bridge $B$ of $G$ we have $|a(B)| \leq 3$.

Note that the sole purpose of $C \cap D=\emptyset$ is to ensure that no $(P \cup Q)$-bridge $B$ of $G$ exists such that both $(B-a(B)) \cap C \neq \emptyset$ and $(B-a(B)) \cap D \neq \emptyset$.

Proof of Lemma 3.3. Let $H$ be the block of $G-x C y$ containing $D$. If $H \cap(y C x-$ $\{x, y\}) \neq \emptyset$, then we use Lemma 2.1 to find a path $Q$ in $H$ from $u$ to $v$ using a vertex in $H \cap(y C x-\{x, y\})$. Now we modify $x C y$ with respect to $(H, Q)$ to get the desired path $P$ (the note and the remark following Lemma 2.6). Thus we may assume that $H \cap y C x=\emptyset$.

Suppose that $H$ and $y C x-\{x, y\}$ belong to different components of $G-x C y$. Then $G$ has a 2-cut $\{p, q\}$ separating $H$ from $y C x-\{p, q\}$, where $x, p, q$ appear on $C$ in this order. Let $T$ be the $\{p, q\}$-bridge of $G$ containing $y C x$. Let $F$ be the outercycle of $H$. Let $J_{1}, \cdots, J_{m}$ be the $(H \cup x C y)$-bridges of $G$ with an attachment in $H$ such that $J_{1} \cap C, \cdots, J_{m} \cap C$ appear on $C$ in this order. Let $J_{i} \cap H=\left\{r_{i}\right\}$. In $H$ we find a path $Q$ from $u$ to $v$ using a vertex in $r_{m} F r_{1}$ (Lemma 2.1). In $T+p q$ we find a path $P_{1}$ from $x$ to $y$ using $p q$ (Lemma 2.1). Now we modify $p C q$ (with respect to $(H, Q)$ ) to get a path $P_{2}$ (by the note and the remark following Lemma 2.6). It is easy to see that $\left(P_{1}-p q\right) \cup P_{2}$ gives the desired $P$.

Therefore we may assume that $H$ and $y C x-\{x, y\}$ belong to a common component of $G-x C y$. Then $G$ has a 3 -cut $\{p, q, w\}$ with $x, p, q$ appearing on $C$ in this order and $w \in H$ is the cutvertex of $G-x C y$ separating $y C x-\{x, y\}$ from $D$. Let $B$ be the $\{p, q, w\}$-bridge of $G$ containing $y C x$. In $H$ we find a path $Q$ from $u$ to $v$ using $w$ (Lemma 2.1). In $B+p q-w$ we view the facial walk of $B+p q-w$ which bounds the face containing $w$ as the outerwalk and find a path $P_{1}$ from $x$ to $y$ using $p q$ (Lemma 2.1). Finally modifying $p C q$ with respect to $(H, Q)$, we get a path $P_{2}$ (the note and the remark following Lemma 2.6). It is easy to verify that $\left(P_{1}-p q\right) \cup P_{2}$ gives the desired path $P$.

We are now ready to prove our main results on disjoint paths.

Theorem 3.4. Let $G$ be a 2-connected plane graph with outercycle $C$ and another facial cycle $D$ disjoint from $C$. Let $x, y \in V(C)$ and $u, v \in V(D)$ be four distinct vertices. Suppose that (1) any simple closed curve in the plane separating $C$ from $D$ intersects $G$ at least 7 times, and (2) any simple curve in the plane from $C$ to $D$ intersects $G$ at least 8 times. Then $G$ has two disjoint paths $P$ and $Q$ with $P$ from $x$ to $y$ and $Q$ from $u$ to $v$ such that for every $(P \cup Q)$-bridge $B$ of $G$, (i) $|a(B)| \leq 4$, and (ii) $|a(B)| \leq 2$ if $(B-a(B)) \cap(C \cup D) \neq \emptyset$.

Proof. Let $C^{\prime}$ be the outercycle of the block of $G-C$ containing $D$, and let $G^{\prime}$ be the block of $G-(C \cup D)$ containing $C^{\prime}$. Let $D^{\prime}$ be the facial cycle of $G^{\prime}$ (other than $C^{\prime}$ ) which is not a facial cycle of $G$. Our strategy is to find vertices $x^{\prime}, y^{\prime} \in C^{\prime}$ and $u^{\prime}, v^{\prime} \in D^{\prime}$ (and if necessary, we go to $G^{\prime}-\left(C^{\prime} \cup D^{\prime}\right)$ ) and to find paths $P^{\prime}$ and $Q^{\prime}$ in $G^{\prime}$ (as in Lemma 3.3) with $P^{\prime}$ from $x^{\prime}$ to $y^{\prime}$ and $Q^{\prime}$ from $u^{\prime}$ to $v^{\prime}$, and then 
to extend them to our desired paths in $G$ by "modifying" $C$ and $D$. Because of symmetry we only treat the " $C$-side", the " $D$-side" can be treated in the same way.

Let $x_{1}^{\prime}, \cdots, x_{m}^{\prime} \in C^{\prime}$ (in this clockwise order on $\left.C^{\prime}\right)$ be the attachments of $\left(G^{\prime} \cup\right.$ $C$ )-bridges of $G$. Let $s_{i} C t_{i}$ be the maximal subpath of $C$ such that every $\left(G^{\prime} \cup\right.$ $C)$-bridge of $G$ with $x_{i}^{\prime}$ as an attachment has all of its attachments in $s_{i} C t_{i} \cup\left\{x_{i}^{\prime}\right\}$ and that $s_{i} C t_{i}-\left\{s_{i}, t_{i}\right\}$ contains no attachments of $\left(G^{\prime} \cup C\right)$-bridges of $G$ with $x_{j}^{\prime}$ as an attachment for any $j \neq i$. Let $J_{i}$ be the union of $s_{i} C t_{i}$ and all $\left(G^{\prime} \cup C\right)$-bridges of $G$ with attachments in $s_{i} C t_{i} \cup\left\{x_{i}^{\prime}\right\}$. Let $T_{i}$ be the union of $s_{i} C s_{i+1}$ and all the $\left(G^{\prime} \cup C\right)$-bridges of $G$ with attachments contained in $s_{i} C s_{i+1} \cup\left\{x_{i}^{\prime}\right\}$.

We may assume $x \in s_{k} C s_{k+1}-\left\{s_{k}\right\}$, otherwise $s_{i}=x$ for each $i$ and so there would be some $x_{j}^{\prime}$ such that a simple closed curve separating $C$ from $D$ intersects $G$ only at $x_{j}^{\prime}$ and $x$. Similarly let $y \in s_{l} C s_{l+1}-\left\{s_{l}\right\}$. By a similar argument, we may also assume that $x \in t_{k^{\prime}-1} C t_{k^{\prime}}-\left\{t_{k^{\prime}}\right\}$ and $y \in t_{l^{\prime}-1} C t_{l^{\prime}}-\left\{t_{l^{\prime}}\right\}$.

In the case that $k=l$ and $k^{\prime}=l^{\prime}$ it is not difficult to see that $x, y \in s_{k} C t_{k}-$ $\left\{s_{k}, t_{k}\right\}$ or $x, y \in t_{k} C s_{k+1}$ for some $k$. We may assume that $s_{k}, x, y$ are on $C$ in this clockwise order.

The rest of the proof is roughly divided into three stages. First we describe $x^{\prime}, y^{\prime}, u^{\prime}$ and $v^{\prime}$. Then we find paths $P^{\prime}$ and $Q^{\prime}$ with $P^{\prime}$ from $x^{\prime}$ to $y^{\prime}$ and $Q^{\prime}$ from $u^{\prime}$ to $v^{\prime}$. In the last stage we extend $P^{\prime}$ and $Q^{\prime}$ to $P$ and $Q$, respectively.

Stage 1. This stage describes $x^{\prime}, y^{\prime}, u^{\prime}, v^{\prime}$. We distinguish three cases: (a), (b) and (c).

(a) $k \neq l$, or $k^{\prime} \neq l^{\prime}$, or $x, y \in t_{k} C s_{k+1}$ for some $k$.

If $k \neq l$, then let $x^{\prime}=x_{k}^{\prime}$ and $y^{\prime}=x_{l}^{\prime}$. If $k^{\prime} \neq l^{\prime}$, then let $x^{\prime}=x_{k^{\prime}}^{\prime}$ and $y^{\prime}=x_{l^{\prime}}^{\prime}$. If $x, y \in t_{k} C s_{k+1}$, then we may select $x^{\prime}=x_{k}^{\prime}$ and $y^{\prime}=x_{p}^{\prime}$, where $x_{p}^{\prime} C^{\prime} x_{k}^{\prime}$ is shortest with $s_{p} \neq t_{k}$. Note that such $x_{p}^{\prime}$ exists, otherwise there would be a simple closed curve in the plane separating $C$ from $D$ intersecting $G$ only at $s_{k}$, contradicting (1).

Now the remaining cases will be for $x, y \in s_{k} C t_{k}-\left\{s_{k}, t_{k}\right\}$. It is clear that $s_{k} C t_{k}$ is contained in a block $J$ of $J_{k}$. Let $z=x_{k}^{\prime}$ if $J=J_{k}$; otherwise let $z \in J$ be the cutvertex of $J_{k}$ separating $x_{k}^{\prime}$ from $s_{k} C t_{k}$.

(b) There are two disjoint paths $X$ and $Y$ in $J$ with $X$ from $x$ to $z$ using $s_{k}$ and $Y$ from $y$ to $t_{k}$ (or symmetrically, $X$ from $x$ to $s_{k}$ and $Y$ from $y$ to $z$ using $t_{k}$ ) such that for every $(X \cup Y)$-bridge $B$ of $J$, (i) $|a(B)| \leq 4$, and (ii) $|a(B)| \leq 2$ if $(B-a(B)) \cap s_{k} C t_{k} \neq \emptyset$.

In this case, we let $x^{\prime}=x_{k}^{\prime}$ and $y^{\prime}=x_{p}^{\prime}$, where $x_{p}^{\prime} C^{\prime} x_{k}^{\prime}$ is shortest (or $x_{k}^{\prime} C^{\prime} x_{p}^{\prime}$ is shortest) such that $s_{p} \neq s_{k}$ (or $t_{p} \neq t_{k}$ ). Note that such a vertex $x_{p}^{\prime}$ always exists, otherwise there would be a simple closed curve in the plane separating $C$ from $D$ which intersects $G$ only at $s_{k}, x_{k}^{\prime}\left(\right.$ or $\left.t_{k}, x_{k}^{\prime}\right)$, contradicting (1).

(c) These two paths $X$ and $Y$ in (b) do not exist.

By Lemma 3.2, $J$ contains two disjoint paths $V$ and $W$ with $V$ from $x$ to $s_{k}$ using $z$ and $W$ from $y$ to $t_{k}$ such that for every $(V \cup W)$-bridge $B$ of $J$, (i) $|a(B)| \leq 4$, and (ii) $|a(B)| \leq 2$ if $(B-a(B)) \cap s_{k} C t_{k} \neq \emptyset$.

By condition (2) let $H$ be the block of $G^{\prime}-z$ containing $D^{\prime}$ and let $E$ be the outercycle of $H$. By (1), $H \cap C^{\prime} \neq \emptyset$. If $z \neq x_{k}^{\prime}$, then $E=C^{\prime}$ and in this case let $z_{1}=z_{2}=x_{k}^{\prime}$. If $z=x_{k}^{\prime}$, then let $z_{1}, z_{2} \in C^{\prime}$ be the attachments of $(H \cup\{z\})$-bridges of $G^{\prime}$ such that $z_{1}, z, z_{2}$ appear on $C^{\prime}$ in this order. Note that $z_{1} \neq z_{2}$, for otherwise there would be a simple closed curve in the plane separating 
$C$ from $D$ which intersects $G$ only at $z_{1}=z_{2}$ and $x_{k}^{\prime}=z$, contradicting (1). Also note that $z_{2} C^{\prime} z_{1}=z_{2} E z_{1}=E \cap C^{\prime}$. Let $N$ be the block of $H-E$ containing $D^{\prime}$, and let $F$ be the outercycle of $N$. Let $w_{1}, \cdots, w_{n} \in F$ (in this order on $F$ ) be the attachments of $(N \cup E)$-bridges of $H$. We define $s_{i}^{\prime} E t_{i}^{\prime}, J_{i}^{\prime}$ and $T_{i}^{\prime}$ in $H$ with respect to $w_{i}, E$ and $F$ much as we defined $s_{i} C t_{i}, J_{i}$ and $T_{i}$ in $G$ with respect to $x_{i}^{\prime}, C$ and $C^{\prime}$.

Claim 1. We can find $x_{a}^{\prime}, x_{b}^{\prime} \in z_{2} C^{\prime} z_{1}-\left\{z_{1}, z_{2}\right\}$ with $z_{2}, x_{a}^{\prime}, x_{b}^{\prime}$ in this order on $C^{\prime}$ such that (i) $s_{a} \neq t_{b}$ and $J_{i} \cap C=\left\{s_{a}=t_{a}\right\}$ for all $x_{i}^{\prime} \in x_{a}^{\prime} E x_{b}^{\prime}-\left\{x_{a}^{\prime}, x_{b}^{\prime}\right\}$ or $J_{i} \cap C=\left\{s_{b}=t_{b}\right\}$ for all $x_{i}^{\prime} \in x_{a}^{\prime} E x_{b}^{\prime}-\left\{x_{a}^{\prime}, x_{b}^{\prime}\right\}$, and (ii) no $w_{c} \in F$ exists with $x_{a}^{\prime}, x_{b}^{\prime} \in s_{c}^{\prime} E t_{c}^{\prime}-\left\{s_{c}^{\prime}, t_{c}^{\prime}\right\}$.

Proof of Claim 1. We first prove that we can select $x_{a}^{\prime}$ and $x_{b}^{\prime}$ satisfying (i). If $x_{i}^{\prime} \notin z_{2} C^{\prime} z_{1}-\left\{z_{1}, z_{2}\right\}$ for every $i$, then there is a simple closed curve in the plane separating $C$ from $D$ which only intersects $G$ at $z_{1}, z_{2}$ and $x_{k}^{\prime}$, a contradiction. If $z_{2} C^{\prime} z_{1}-\left\{z_{1}, z_{2}\right\}$ contains exactly one $x_{i}^{\prime}$, then there would be a simple closed curve in the plane separating $C$ from $D$ which only intersects $G$ at $x_{i}^{\prime}, z_{1}, z_{2}$ and $x_{k}^{\prime}$. So we may assume that $z_{2} C^{\prime} z_{1}-\left\{z_{1}, z_{2}\right\}$ contains at least two $x_{i}^{\prime}$. Then this pair $x_{a}^{\prime}$ and $x_{b}^{\prime}$ can be selected unless $s_{i}=t_{i}=s$ for every $x_{i}^{\prime} \in z_{2} C^{\prime} z_{1}-\left\{z_{1}, z_{2}\right\}$. But then there is a simple closed curve in the plane separating $C$ from $D$ which intersects $G$ only at $z_{1}, z_{2}, s$ and $x_{k}^{\prime}$, a contradiction.

Now suppose that (ii) is violated for all of those $x_{a}^{\prime}, x_{b}^{\prime}$ satisfying (i), that is, there is a $w_{c}$ such that $x_{a}^{\prime}, x_{b}^{\prime} \in s_{c}^{\prime} E t_{c}^{\prime}-\left\{s_{c}^{\prime}, t_{c}^{\prime}\right\}$.

Note that $s_{c}^{\prime}, z_{2}, z_{1}, t_{c}^{\prime}$ cannot appear on $E$ in this order, otherwise there would be a simple closed curve in the plane separating $C$ from $D$ which intersects $G$ only at $w_{c}, s_{c}^{\prime}, t_{c}^{\prime}$ and $x_{k}^{\prime}$. We now show that $z_{1}, z_{2}$ cannot both be contained in $s_{c}^{\prime} C^{\prime} t_{c}^{\prime}$. Suppose $z_{1}, z_{2} \in s_{c}^{\prime} C^{\prime} t_{c}^{\prime}$. Then there is $x_{r}^{\prime} \in t_{c}^{\prime} C^{\prime} s_{c}^{\prime}$, otherwise there would be a simple closed curve separating $C$ from $D$ which intersects $G$ only at $s_{c}^{\prime}, t_{c}^{\prime}$ and $w_{c}$. We may select $x_{r}^{\prime}$ so that $x_{b}^{\prime} C^{\prime} x_{r}^{\prime}$ is shortest if $x_{a}^{\prime}, x_{b}^{\prime} \in z_{2} C^{\prime} t_{c}^{\prime}$ or $x_{r}^{\prime} C^{\prime} x_{a}^{\prime}$ is shortest if $x_{a}^{\prime}, x_{b}^{\prime} \in s_{c}^{\prime} C^{\prime} z_{1}$. It is easy to see that there is a $x_{d}^{\prime} \in x_{a}^{\prime} C^{\prime} x_{r}^{\prime}$ if $x_{a}^{\prime}, x_{b}^{\prime} \in z_{2} C^{\prime} t_{c}^{\prime}$ or $x_{d}^{\prime} \in x_{r}^{\prime} C^{\prime} x_{b}^{\prime}$ if $x_{a}^{\prime}, x_{b}^{\prime} \in s_{c}^{\prime} C^{\prime} z_{1}$, such that $x_{d}^{\prime}$ and $x_{r}^{\prime}$ form the desired pair (using the fact that $x_{a}^{\prime}$ and $x_{b}^{\prime}$ satisfy (i)), a contradiction.

Therefore we can select $x_{r}^{\prime} \in z_{2} C^{\prime} s_{c}^{\prime}-\left\{z_{2}\right\}$ (or $x_{r}^{\prime} \in t_{c}^{\prime} C^{\prime} z_{1}-\left\{z_{1}\right\}$ ) such that $x_{r}^{\prime} C^{\prime} x_{a}^{\prime}$ (or $x_{b}^{\prime} C^{\prime} x_{r}^{\prime}$ ) is shortest; otherwise there would be a simple closed curve in the plane separating $C$ from $D$ which intersects $G$ only (possibly) at $s_{c}^{\prime}, t_{c}^{\prime}, w_{c}, z_{1}, z_{2}$ and $x_{k}^{\prime}$. It is now easy to check (by using the fact that $x_{a}^{\prime}$ and $x_{b}^{\prime}$ satisfy (i)) that we can find $x_{d}^{\prime} \in x_{r}^{\prime} C^{\prime} x_{b}^{\prime}$ (or $x_{d}^{\prime} \in x_{a}^{\prime} C^{\prime} x_{r}^{\prime}$ ) such that $x_{r}^{\prime}$ and $x_{d}^{\prime}$ give the desired pair, a contradiction. Hence Claim 1 follows.

By (ii) of Claim 1, we can find $w_{p} \in F$ such that $x_{a}^{\prime}, x_{b}^{\prime} \in t_{p}^{\prime} C^{\prime} s_{p+1}^{\prime}$ or we can find $w_{p}, w_{q} \in F$ with $w_{p} \neq w_{q}$ such that $x_{a}^{\prime} \in s_{p}^{\prime} E s_{p+1}^{\prime}-\left\{s_{p}^{\prime}\right\}$ and $x_{b}^{\prime} \in s_{q}^{\prime} E s_{q+1}^{\prime}-\left\{s_{q}^{\prime}\right\}$ or (symmetrically) $x_{a}^{\prime} \in t_{p-1}^{\prime} E t_{p}^{\prime}-\left\{t_{p}^{\prime}\right\}$ and $x_{b}^{\prime} \in t_{q-1}^{\prime} E t_{q}^{\prime}-\left\{t_{q}^{\prime}\right\}$. In the former case we let $y^{\prime}=w_{p}$ and $x^{\prime}=w_{q}$, where $w_{q} F w_{p}$ is shortest with $s_{q}^{\prime} \neq t_{p}^{\prime}$. In the latter case we let $x^{\prime}=w_{p}$ and $y^{\prime}=w_{q}$.

By a similar treatment we have $D^{\prime}, u^{\prime}, v^{\prime}$, and $L$ at the " $D$-side" precisely the same as $C^{\prime}, x^{\prime}, y^{\prime}$, and $F$ at the " $C$-side". By (2) we see that $F \cap L=\emptyset$.

Stage 2. We now find $P^{\prime}$ and $Q^{\prime}$. Let $H^{\prime}$ be the plane subgraph of $G$ such that: $H^{\prime}=G^{\prime}$ if $x^{\prime}, y^{\prime} \in C^{\prime}$ and $u^{\prime}, v^{\prime} \in D^{\prime} ; H^{\prime}$ is the subgraph of $G$ between $C^{\prime}$ and $L$ (i.e., the subgraph of $G$ contained in the closed annulus bounded by $C^{\prime}$ and $L$ ) if $x^{\prime}, y^{\prime} \in C^{\prime}$ and $u^{\prime}, v^{\prime} \in L ; H^{\prime}$ is the subgraph of $G$ between $F$ and $D^{\prime}$ if $x^{\prime}, y^{\prime} \in F$ 
and $u^{\prime}, v^{\prime} \in D^{\prime}$; or $H^{\prime}$ is the subgraph of $G$ between $F$ and $L$ if $x^{\prime}, y^{\prime} \in F$ and $u^{\prime}, v^{\prime} \in L$. For convenience we use $F^{\prime}$ and $L^{\prime}$ to denote the two facial cycles of $H^{\prime}$ which are not facial cycles of $G$, where $F^{\prime}$ is the outercycle. Hence $F^{\prime}=F$ or $F^{\prime}=C^{\prime}$ and $L^{\prime}=L$ or $L^{\prime}=D^{\prime}$.

By (2) we have $F^{\prime} \cap L^{\prime}=\emptyset$. Hence by Lemma 3.3 we can find two disjoint paths $P^{\prime}$ and $Q^{\prime}$ in $H^{\prime}$ with $P^{\prime}$ from $x^{\prime}$ to $y^{\prime}$ and $Q^{\prime}$ from $u^{\prime}$ to $v^{\prime}$ such that for every $\left(P^{\prime} \cup Q^{\prime}\right)$-bridge $B$ of $H^{\prime}$ we have that $|a(B)| \leq 3$. Note that if $(B-a(B)) \cap F^{\prime} \neq \emptyset$, then $(B-a(B)) \cap L^{\prime}=\emptyset$.

Stage 3. We now extend $P^{\prime}$ to a path from $x$ to $y$ and $Q^{\prime}$ to a path from $u$ to $v$. By symmetry we only show how to extend $P^{\prime}$. Suppose first that $F^{\prime}=C^{\prime}$. Then we have (a) or (b). For (a) we may first assume that $x \in s_{k} C s_{k+1}-\left\{s_{k}\right\}$ and $y \in s_{l} C s_{l+1}-\left\{s_{l}\right\}$ with $x_{k}^{\prime} \neq x_{l}^{\prime}$. (Note that the case that $x \in t_{k^{\prime}-1} C t_{k^{\prime}}-\left\{t_{k^{\prime}}\right\}$ and $y \in t_{l^{\prime}-1} C t_{l^{\prime}}-\left\{t_{l^{\prime}}\right\}$ with $x_{k^{\prime}}^{\prime} \neq x_{l^{\prime}}^{\prime}$ can be treated in the same way.) In this case $x^{\prime}=x_{k}^{\prime}$ and $y^{\prime}=x_{l}^{\prime}$. In $T_{k}+s_{k+1} x_{k}^{\prime}$ we find a path $Q_{k}$ from $x$ to $s_{k}$ using $s_{k+1} x_{k}^{\prime}$ (Lemma 2.1). In $T_{l}+s_{l+1} x_{l}^{\prime}$ we find a path $Q_{l}$ from $y$ to $s_{l}$ using $s_{l+1} x_{l}^{\prime}$ (Lemma 2.1). We then modify $s_{k+1} C s_{l}$ and $s_{l+1} C s_{k}$ (both with respect to $\left(H^{\prime}, P^{\prime} \cup Q^{\prime}\right)$ ) to get two paths $R_{1}$ and $R_{2}$. It is easy to see that $P=P^{\prime} \cup\left(Q_{k}-s_{k+1} x_{k}^{\prime}\right) \cup\left(Q_{l}-s_{l+1} x_{l}^{\prime}\right) \cup R_{1} \cup R_{2}$ is as desired. So we may assume that $x, y \in t_{k} C s_{k+1}$ and $s_{k}, x, y$ on $C$ in this order. In this case $x^{\prime}=x_{k}^{\prime}$ and $y^{\prime}=x_{p}^{\prime}$. In $J_{p}$ we find a path $Q_{p}$ from $x_{p}^{\prime}$ to $s_{p}$ using $t_{p}$ if $t_{p} \neq t_{k}$ (Lemma 2.1), otherwise not using $t_{p}$ (Lemma 2.5). In $J_{k}$ we find a path $Q_{k}$ from $x_{k}^{\prime}$ to $t_{k}$ using $s_{k}$ if $s_{k} \notin Q_{p}$ (Lemma 2.1), otherwise not using $s_{k}$ (Lemma 2.5). In the union of the edge $s_{k+1} t_{k}$ and the $\left\{s_{k+1}, t_{k}\right\}$-bridge of $G$ containing $x, y$ we find a path $R_{k}$ from $x$ to $y$ using $s_{k+1} t_{k}$ (Lemma 2.1). Finally we modify $s_{k+1} C s_{p}$ with respect to $\left(H^{\prime}, P^{\prime} \cup Q^{\prime}\right)$ to get a path $R$. Then $P=P^{\prime} \cup Q_{k} \cup Q_{p} \cup\left(R_{k}-s_{k+1} t_{k}\right) \cup R$ is the desired path.

For (b) we use $X$ and $Y$ and $x^{\prime}=x_{k}^{\prime}$ and $y^{\prime}=x_{p}^{\prime}$. By symmetry we only consider the case when $X$ is from $x$ to $z$ using $s_{k}$. In $J_{p}$ we find a path $Q_{p}$ from $x_{p}^{\prime}$ to $s_{p}$ using $t_{p}$ if $t_{p} \neq s_{k}$ (Lemma 2.1), otherwise not using $t_{p}$ (Lemma 2.5). Let $Z=\left\{x_{k}^{\prime}\right\}$ if $z=x_{k}^{\prime}$, otherwise let $Z$ be a path (Lemma 2.1) from $z$ to $x_{k}^{\prime}$ in the $z$-bridge of $J_{k}$ containing $x_{k}^{\prime}$. Now we modify $t_{k} C s_{p}$ with respect to $\left(H^{\prime}, P^{\prime} \cup Q^{\prime}\right)$ to get a path $R$. Hence $P=P^{\prime} \cup X \cup Y \cup Z \cup Q_{p} \cup R$ is as desired.

Thus we have (c), i.e., $F^{\prime}=F$. We first extend $P^{\prime}$ to a path $P^{*}$ in $H$ from $x_{a}^{\prime}$ to $x_{b}^{\prime}$. Suppose first $x_{a}^{\prime} \in s_{p}^{\prime} E s_{p+1}^{\prime}-\left\{s_{p}^{\prime}\right\}$ and $x_{b}^{\prime} \in s_{q}^{\prime} E s_{q+1}^{\prime}-\left\{s_{q}^{\prime}\right\}$, where $w_{p} \neq w_{q}$. (The case that $x_{a}^{\prime} \in t_{p-1}^{\prime} E t_{p}^{\prime}-\left\{t_{p}^{\prime}\right\}$ and $x_{b}^{\prime} \in t_{q-1}^{\prime} E t_{q}^{\prime}-\left\{t_{q}^{\prime}\right\}$ with $w_{p} \neq w_{q}$ can be treated in the same way.) In this case $x^{\prime}=w_{p}$ and $y^{\prime}=w_{q}$. In $T_{p}^{\prime}+s_{p+1}^{\prime} w_{p}$ we find a path $Q_{p}^{\prime}$ from $x_{a}^{\prime}$ to $s_{p}^{\prime}$ using $s_{p+1}^{\prime} w_{p}$. In $T_{q}^{\prime}+s_{q+1}^{\prime} w_{q}$ we find a path $Q_{q}^{\prime}$ from $x_{b}^{\prime}$ to $s_{q}^{\prime}$ using $s_{q+1}^{\prime} w_{q}$. We modify $s_{q+1}^{\prime} E s_{p}^{\prime}$ and $s_{p+1}^{\prime} E s_{q}^{\prime}$ (both with respect to $\left(H^{\prime}, P^{\prime} \cup Q^{\prime}\right)$ ) to get two paths $R_{1}^{\prime}$ and $R_{2}^{\prime}$. Let $P^{*}=P^{\prime} \cup\left(Q_{p}^{\prime}-s_{p+1}^{\prime} w_{p}\right) \cup\left(Q_{q}^{\prime}-s_{q+1}^{\prime} w_{q}\right) \cup R_{1}^{\prime} \cup R_{2}^{\prime}$ (a path from $x_{a}^{\prime}$ to $x_{b}^{\prime}$ ).

Hence by the above claim and by symmetry we may assume that $t_{p}^{\prime}, x_{a}^{\prime}, x_{b}^{\prime}, s_{p+1}^{\prime}$ are on $C^{\prime}$ in this order. In this case we have $x^{\prime}=w_{q}$ and $y^{\prime}=w_{p}$. In $J_{q}^{\prime}$ we find a path $Q_{q}^{\prime}$ from $w_{q}$ to $s_{q}^{\prime}$ using $t_{q}^{\prime}$ (Lemma 2.1) if $t_{q}^{\prime} \neq t_{p}^{\prime}$, otherwise not using $t_{q}^{\prime}$ (Lemma 2.5). In $J_{p}^{\prime}$ we find a path $Q_{p}^{\prime}$ from $w_{p}$ to $t_{p}^{\prime}$ using $s_{p}^{\prime}$ if $s_{p}^{\prime} \notin Q_{q}^{\prime}$ (Lemma 2.1), otherwise not using $s_{p}^{\prime}$ (Lemma 2.5). In the union of the edge $s_{p+1}^{\prime} t_{p}^{\prime}$ and the $\left\{t_{p}^{\prime}, s_{p+1}^{\prime}\right\}$-bridge of $H$ containing $x_{a}^{\prime} C^{\prime} x_{b}^{\prime}$ we find a path $R_{p}^{\prime}$ from $x_{a}^{\prime}$ to $x_{b}^{\prime}$ using $s_{p+1}^{\prime} t_{p}^{\prime}$ (Lemma 2.1). Finally we modify $s_{p+1}^{\prime} E s_{q}^{\prime}$ with respect to $\left(H^{\prime}, P^{\prime} \cup Q^{\prime}\right)$ to get a path $R^{\prime}$. Let $P^{*}=P^{\prime} \cup Q_{p}^{\prime} \cup Q_{q}^{\prime} \cup\left(R_{p}^{\prime}-s_{p+1}^{\prime} t_{p}^{\prime}\right) \cup R^{\prime}$. Note that $|a(B)| \leq 2$ if the $P^{*}$-bridge $B$ of $H$ has $(B-a(B)) \cap E \neq \emptyset$. 
Suppose that $z_{1}, z_{2}$ do not both belong to $B-a(B)$ for any $P^{*}$-bridge $B$ of $H$. In this case either $z=x_{k}^{\prime}$ and $z_{1} \neq z_{2}$ or $z \neq x_{k}^{\prime}$ and $x_{k}^{\prime} \in P^{*}$. We find a path $Q_{a}$ in $J_{a}$ from $x_{a}^{\prime}$ to $s_{a}$ using $t_{a}$ if $t_{a} \neq t_{b}$ (Lemma 2.1), otherwise not using $t_{a}$ (Lemma 2.5). In $J_{b}$ we find a path $Q_{b}$ from $x_{b}^{\prime}$ to $t_{b}$ using $s_{b}$ if $s_{b} \notin Q_{a}$ (Lemma 2.1), otherwise not using $s_{b}$ (Lemma 2.5). Finally we modify $t_{k} C s_{a}$ and $t_{b} C s_{k}$ (both with respect to $\left.\left(G^{\prime}, P^{*} \cup Q^{\prime} \cup\left\{x_{k}^{\prime}\right\}\right)\right)$ to get two paths $R_{1}$ and $R_{2}$. Then $P=P^{*} \cup V \cup W \cup Q_{a} \cup Q_{b} \cup R_{1} \cup R_{2}$ is as desired.

Hence we may assume that $z_{1}, z_{2} \in B-a(B)$ for some $P^{*}$-bridge $B$ of $H$. We may also assume that both $x_{k-1}^{\prime}, x_{k+1}^{\prime} \in B-a(B)$; otherwise we can directly modify $C$ exactly the same way as in the previous paragraph. Let $a(B)=\left\{v_{1}, v_{2}\right\}$ such that $v_{1}, z_{1}, z_{2}, v_{2}$ appear on $C^{\prime}$ in this order. We select $P^{*}$ so that $B$ is minimal.

Claim 2. There is some $w_{d} \in F$ such that $z_{1}, z_{2} \in s_{d}^{\prime} E t_{d}^{\prime}-\left\{s_{d}^{\prime}, t_{d}^{\prime}\right\}$ or $z_{1}, z_{2} \in$ $t_{d}^{\prime} E s_{d+1}^{\prime}-\left\{t_{d}^{\prime}, s_{d+1}^{\prime}\right\}$.

Proof of Claim 2. Suppose this claim is not true. By Lemma 2.8 we have $z_{1}, z_{2} \in$ $s_{q}^{\prime} E s_{p+1}^{\prime}$ when $x_{a}^{\prime}, x_{b}^{\prime} \in t_{p}^{\prime} C^{\prime} s_{p+1}^{\prime}$ (since in this case we modify $s_{p+1}^{\prime} E s_{q}^{\prime}$ ), and $z_{1}, z_{2} \in$ $T_{p}^{\prime}$ or $z_{1}, z_{2} \in T_{q}^{\prime}$ when $x_{a}^{\prime} \in T_{p}^{\prime}$ and $x_{q}^{\prime} \in T_{q}^{\prime}$ with $w_{p} \neq w_{q}$ (since in this case we modify $s_{q+1}^{\prime} E s_{p}^{\prime}$ and $s_{p+1}^{\prime} E s_{q}^{\prime}$ ); otherwise we can modify $E$ by Lemma 2.8 so that there is no $P^{*}$-bridge $B$ of $H$ with $z_{1}, z_{2} \in B-a(B)$. If $z_{1}, z_{2} \in s_{q}^{\prime} E s_{p+1}^{\prime}$, then $z_{1}, z_{2} \in J_{q}^{\prime}$ or $z_{1}, z_{2} \in J_{p}^{\prime}$ (otherwise $z_{1}, z_{2} \in t_{q}^{\prime} E s_{q+1}^{\prime}-\left\{t_{q}^{\prime}, s_{q+1}^{\prime}\right\}$ ). By symmetry let $z_{1}, z_{2} \in J_{q}^{\prime}$. Then $\left\{z_{1}, z_{2}\right\} \cap\left\{s_{q}^{\prime}, t_{q}^{\prime}\right\} \neq \emptyset$ (otherwise $\left(Q_{q}^{\prime} \cup Q_{p}^{\prime}\right) \cap\left\{z_{1}, z_{2}\right\} \neq \emptyset$ ), but then $z_{1}, z_{2} \in s_{q}^{\prime} E t_{q}^{\prime}-\left\{s_{q}^{\prime}, t_{q}^{\prime}\right\}$, a contradiction.

So by symmetry, let $z_{1}, z_{2} \in T_{p}^{\prime}$. Moreover, we may assume that $z_{1} \in s_{p}^{\prime} E t_{p}^{\prime}$ and $z_{2} \in t_{p}^{\prime} E s_{p+1}^{\prime}$ (otherwise the claim would follow). In this case we can change the part $Q_{p}^{\prime}-s_{p+1}^{\prime} w_{p}$ of $P^{*}$ so that no $P^{*}$-bridge $B$ of $H$ exists with $z_{1}, z_{2} \in B-a(B)$. First note that $x_{a}^{\prime} \notin s_{p}^{\prime} E t_{p}^{\prime}$, otherwise $z_{1}$ and $z_{2}$ do not belong to the same $P^{*}$-bridge of $H$, a contradiction. So $x_{a}^{\prime} \in t_{p}^{\prime} E s_{p+1}^{\prime}-\left\{t_{p}^{\prime}\right\}$. Then we find a path in $J_{p}^{\prime}$ from $w_{p}$ to $s_{p}^{\prime}$ using $t_{p}^{\prime}$ (Lemma 2.1). In the union of $t_{p}^{\prime} E s_{p+1}^{\prime}$ and all $(E \cup N)$-bridges of $H$ with attachments contained in $t_{p}^{\prime} E s_{p+1}^{\prime}$, we find a path from $x_{a}^{\prime}$ to $s_{p+1}^{\prime}$ not using $t_{p}^{\prime}$ (Lemma 2.5). Now the union of these two paths is used to replace the $Q_{p}^{\prime}-s_{p+1}^{\prime} w_{p}$ part of $P^{*}$. Clearly no $P^{*}$-bridge $B$ of $H$ exists such that $B-a(B)$ contains both $z_{1}$ and $z_{2}$, a contradiction.

By Claim 2, we have two cases. Note that we may change $x^{\prime}$ and $y^{\prime}$ in the following arguments. Also note that since $|a(B)|=2, v_{1}, v_{2} \in s_{d}^{\prime} E t_{d}^{\prime}$ or $v_{1}, v_{2} \in$ $t_{d}^{\prime} E s_{d+1}^{\prime}$ according as $z_{1}, z_{2} \in s_{d}^{\prime} E t_{d}^{\prime}-\left\{s_{d}^{\prime}, t_{d}^{\prime}\right\}$ or $z_{1}, z_{2} \in t_{d}^{\prime} E s_{d+1}^{\prime}-\left\{t_{d}^{\prime}, s_{d+1}^{\prime}\right\}$.

(I) $z_{1}, z_{2} \in s_{d}^{\prime} E t_{d}^{\prime}-\left\{s_{d}^{\prime}, t_{d}^{\prime}\right\}$.

By (1) we may assume that $s_{d}^{\prime}, z_{1}, z_{2}$ are on $E$ in this order. Note that $x_{k-1}, x_{k+1}$ $\in B-a(B)$. Hence either there are $x_{g}^{\prime} \in x_{k}^{\prime} C^{\prime} t_{d}^{\prime}-\left\{x_{k}^{\prime}, t_{d}^{\prime}\right\}$ and $x_{h}^{\prime} \in t_{d}^{\prime} C^{\prime} s_{d}^{\prime}-\left\{s_{d}^{\prime}\right\}$ such that $s_{g} \neq t_{h}$ and $s_{i}=t_{i}=s_{g}$ for all $x_{i}^{\prime} \in x_{g}^{\prime} C^{\prime} x_{h}^{\prime}-\left\{x_{g}^{\prime}, x_{h}^{\prime}\right\}$ (or $s_{i}=t_{i}=t_{h}$ for all $\left.x_{i}^{\prime} \in x_{g}^{\prime} C^{\prime} x_{h}^{\prime}-\left\{x_{g}^{\prime}, x_{h}^{\prime}\right\}\right)$ or there are $x_{g}^{\prime} \in s_{d}^{\prime} C^{\prime} x_{k}^{\prime}-\left\{x_{k}^{\prime}, s_{d}^{\prime}\right\}$ and $x_{h}^{\prime} \in$ $t_{d}^{\prime} C^{\prime} s_{d}^{\prime}-\left\{t_{d}^{\prime}\right\}$ such that $s_{h} \neq t_{g}$ and that $s_{i}=t_{i}=t_{g}$ for all $x_{i}^{\prime} \in x_{h}^{\prime} C^{\prime} x_{g}^{\prime}-\left\{x_{h}^{\prime}, x_{g}^{\prime}\right\}$ (or $s_{i}=t_{i}=s_{h}$ for all $x_{i}^{\prime} \in x_{h}^{\prime} C^{\prime} x_{g}^{\prime}-\left\{x_{g}^{\prime}, x_{h}^{\prime}\right\}$ ). For otherwise we deduce that $s_{i}=t_{i}=w$ for every $x_{i}^{\prime} \in t_{d}^{\prime} C^{\prime} s_{d}^{\prime}$. Thus we have a simple closed curve in the plane separating $C$ from $D$ which intersects $G$ only at $s_{d}^{\prime}, t_{d}^{\prime}, w, w_{d}$, contradicting (1).

Without loss of generality we may assume that $x_{g}^{\prime} \in x_{k}^{\prime} C^{\prime} t_{d}^{\prime}-\left\{x_{k}^{\prime}, t_{d}^{\prime}\right\}$ and $x_{h}^{\prime} \in$ $t_{d}^{\prime} C^{\prime} s_{d}^{\prime}-\left\{s_{d}^{\prime}\right\}$. Note that we may select $w_{r} \in F-w_{d}$ so that $x_{h}^{\prime} \in t_{r-1}^{\prime} C^{\prime} t_{r}^{\prime}-\left\{t_{r}^{\prime}\right\}$, for otherwise $s_{i}^{\prime} E t_{i}^{\prime} \subset t_{d}^{\prime} E x_{h}^{\prime}$ for each $i \neq d$ and so there is a simple closed curve 
in the plane separating $C$ from $D$ which intersects $G$ only at $w_{d}, t_{d}^{\prime}, x_{h}^{\prime}$ (and $s_{g}$ or $t_{h}$ if $s_{g}=t_{g}$ or $s_{h}=t_{h}$ ), violating (1). We now change $x^{\prime}$ and $y^{\prime}$ in $H^{\prime}$ to $w_{d}$ and $w_{r}$, respectively, and use Lemma 3.3 to find the two paths $P^{\prime}$ and $Q^{\prime}$ with $P^{\prime}$ from $x^{\prime}=w_{d}$ to $y^{\prime}=w_{r}$. In $J_{d}^{\prime}+w_{d} s_{d}^{\prime}$ we use Lemma 2.4 to find a path $Q_{d}^{\prime}$ from $x_{g}^{\prime}$ to $t_{d}^{\prime}$ using $w_{d} s_{d}^{\prime}$ and $z_{1}$. (Note that $z_{1}=x_{k}^{\prime}$ if $z \neq x_{k}^{\prime}$.) In the union of $t_{r-1}^{\prime} E t_{r}^{\prime}, w_{r} t_{r-1}^{\prime}$ and all the $(N \cup E)$-bridges of $H$ with all attachments in $t_{r-1}^{\prime} E t_{r}^{\prime} \cup\left\{w_{r}\right\}$, we find a path $Q_{r}^{\prime}$ from $t_{r}^{\prime}$ to $x_{h}^{\prime}$ using $w_{r} t_{r-1}^{\prime}$. Now we modify $t_{d}^{\prime} E t_{r-1}^{\prime}$ and $t_{r}^{\prime} E s_{d}^{\prime}$ (both with respect to $\left(H^{\prime}, P^{\prime} \cup Q^{\prime}\right)$ ) to get two paths $R_{1}^{\prime}$ and $R_{2}^{\prime}$. Let $P^{*}=P^{\prime} \cup\left(Q_{d}^{\prime}-w_{d} s_{d}^{\prime}\right) \cup\left(Q_{r}^{\prime}-w_{r} t_{r-1}^{\prime}\right) \cup R_{1}^{\prime} \cup R_{2}^{\prime}$.

We now extend the path $P^{*}$ to $x$ and $y$. In $J_{g}$ we find a path $Q_{g}$ from $x_{g}^{\prime}$ to $s_{g}$ using $t_{g}$ if $t_{g} \neq t_{h}$ (Lemma 2.1), otherwise not using $t_{g}$ (Lemma 2.5). In $J_{h}$ we find a path $Q_{h}$ from $x_{h}^{\prime}$ to $t_{h}$ using $s_{h}$ if $s_{h} \notin Q_{g}$ (Lemma 2.1), otherwise not using $s_{h}$ (Lemma 2.5). Finally we modify $t_{k} C s_{g}$ and $t_{h} C s_{k}$ (both with respect to $\left.\left(G^{\prime}, P^{*} \cup Q^{\prime}\right)\right)$ to get two paths $R_{1}$ and $R_{2}$. Then $P=P^{*} \cup V \cup W \cup Q_{g} \cup Q_{h} \cup R_{1} \cup R_{2}$ is as desired.

(II) $z_{1}, z_{2} \in t_{d}^{\prime} E s_{d+1}^{\prime}-\left\{t_{d}^{\prime}, s_{d+1}^{\prime}\right\}$.

In this case it is easy to see that $v_{1}=t_{d}^{\prime}$ and $v_{2}=s_{d+1}^{\prime}$. We claim that there is $x_{p}^{\prime} \in t_{d+1}^{\prime} E t_{d}^{\prime}-\left\{t_{d+1}^{\prime}\right\}$ and $x_{q}^{\prime} \in v_{1} C^{\prime} x_{k}^{\prime}-\left\{v_{1}, x_{k}^{\prime}\right\}$ (or $x_{p}^{\prime} \in s_{d+1}^{\prime} E s_{d}^{\prime}-\left\{s_{d}^{\prime}\right\}$ and $\left.x_{q}^{\prime} \in x_{k}^{\prime} C^{\prime} v_{2}-\left\{x_{k}^{\prime}, v_{2}\right\}\right)$ such that $s_{p} \neq t_{q}\left(\right.$ or $\left.t_{p} \neq s_{q}\right)$ and $s_{i}=t_{i}=s_{p}$ for all $x_{i}^{\prime} \in x_{p}^{\prime} C^{\prime} x_{q}^{\prime}-\left\{x_{p}^{\prime}, x_{q}^{\prime}\right\}$ or $s_{i}=t_{i}=t_{q}$ for all $x_{i}^{\prime} \in x_{p}^{\prime} C^{\prime} x_{q}^{\prime}-\left\{x_{p}^{\prime}, x_{q}^{\prime}\right\}$ (or $s_{i}=t_{i}=s_{q}$ for all $x_{i}^{\prime} \in x_{q}^{\prime} C^{\prime} x_{p}^{\prime}-\left\{x_{p}^{\prime}, x_{q}^{\prime}\right\}$ or $s_{i}=t_{i}=t_{p}$ for all $\left.x_{i}^{\prime} \in x_{q}^{\prime} C^{\prime} x_{p}^{\prime}-\left\{x_{p}^{\prime}, x_{q}^{\prime}\right\}\right)$. Otherwise $s_{i}=t_{i}=s$ for each $x_{i}^{\prime} \in v_{1} C^{\prime} x_{k}^{\prime}-\left\{v_{1}, x_{k}^{\prime}\right\}$ and $s_{i}=t_{i}=t$ for each $x_{i}^{\prime} \in x_{k}^{\prime} C^{\prime} v_{2}-\left\{x_{k}^{\prime}, v_{2}\right\}$, and if $x_{i}^{\prime} \in t_{d+1}^{\prime} C^{\prime} s_{d}^{\prime}$ then $s_{i}=t_{i}=s$ or $s_{i}=t_{i}=t$. Hence there would be a simple closed curve in the plane separating $C$ from $D$ which intersects $G$ only at $s, t, v_{1}=t_{d}^{\prime}, v_{2}=s_{d+1}^{\prime}$, contradicting (1). Hence by symmetry we may assume that $x_{p}^{\prime} \in t_{d+1}^{\prime} E t_{d}^{\prime}-\left\{t_{d+1}^{\prime}\right\}$ and $x_{q}^{\prime} \in v_{1} C^{\prime} x_{k}^{\prime}-\left\{v_{1}, x_{k}^{\prime}\right\}$.

Suppose first $x_{p}^{\prime}=v_{1}$. Note that there is a $w_{r} \in w_{d+1} F w_{d}-\left\{w_{d}, w_{d+1}\right\}$, otherwise there would be a simple closed curve in the plane separating $C$ from $D$ which intersects $G$ only at $w_{d}$ and $w_{d+1}$. We can select such $w_{r}$ that $w_{d+1} F w_{r}$ is shortest with $v_{2} \neq t_{r}^{\prime}$, for otherwise there would be a simple closed curve in the plane separating $C$ from $D$ which only intersects $G$ at $w_{d}, v_{2}$. Now in $H^{\prime}$ we replace $x^{\prime}$ and $y^{\prime}$ by $w_{d+1}$ and $w_{r}$ and we find the paths $P^{\prime}$ and $Q^{\prime}$ by Lemma 3.3 with $P^{\prime}$ from $w_{d+1}$ to $w_{r}$.

We extend $P^{\prime}$ to a path $P^{*}$ in $H$ from $x_{p}^{\prime}=v_{1}$ to $x_{q}^{\prime}$. In $J_{d+1}^{\prime}$ we find a path $R_{d+1}^{\prime}$ from $w_{d+1}$ to $v_{2}$ using $t_{d+1}^{\prime}$ if $t_{d+1}^{\prime} \neq t_{r}^{\prime}$ (Lemma 2.1), otherwise not using $t_{d+1}^{\prime}$ (Lemma 2.5). In $J_{r}^{\prime}$ we find a path $R_{r}^{\prime}$ from $w_{r}$ to $t_{r}^{\prime}$ using $s_{r}^{\prime}$ if $s_{r}^{\prime} \notin R_{d+1}^{\prime}$ (Lemma 2.1), otherwise not using $s_{r}^{\prime}$ (Lemma 2.5). In $B-\left\{v_{1}, z\right\}$ we find a path $R^{\prime}$ from $x_{q}^{\prime}$ to $v_{2}$ using $z_{2}$ (Lemma 2.1). (Note that $x_{k}^{\prime}=z_{2}$ if $z \neq x_{k}^{\prime}$.) Now modify $t_{r}^{\prime} E v_{1}$ with respect to $\left(H^{\prime}, P^{\prime} \cup Q^{\prime}\right)$ to get a path to complete $P^{*}$. We now extend $P^{*}$ to $P$. In $J_{p}$ we find a path $R_{p}$ from $v_{1}=x_{p}^{\prime}$ to $s_{p}$ using $t_{p}$ if $t_{p} \neq t_{q}$ (Lemma 2.1 ), otherwise not using $t_{p}$ (Lemma 2.5). In $J_{q}$ we find a path $R_{q}$ from $x_{q}^{\prime}$ to $t_{q}$ using $s_{q}$ if $s_{q} \notin R_{p}$ (Lemma 2.1), otherwise not using $s_{q}$ (Lemma 2.5). Finally we modify $t_{k} C s_{p}$ and $t_{q} C s_{k}$ (both with respect to $\left(G^{\prime}, P^{*} \cup Q^{\prime}\right)$ ) to get two paths $R$ and $S$. Then $P=P^{*} \cup V \cup W \cup R_{p} \cup R_{q} \cup R \cup S$ is the desired path.

Hence we may assume that $x_{p}^{\prime} \neq v_{1}$. We select $w_{r}$ with $t_{r}^{\prime} \in x_{p}^{\prime} E v_{1}-\left\{x_{p}^{\prime}\right\}$ so that $x_{p}^{\prime} E t_{r}^{\prime}$ is shortest (where $w_{r}=w_{d}$ is possible). Now in $H^{\prime}$ we use Lemma 3.3 to find two paths $P^{\prime}$ and $Q^{\prime}$ with $P^{\prime}$ from $x^{\prime}=w_{r}$ to $y^{\prime}=w_{d+1}$. 
We extend $P^{\prime}$ to a path $P^{*}$ in $H$ from $x_{p}^{\prime}$ to $x_{q}^{\prime}$. In $J_{d+1}^{\prime}$ we find a path $R_{d+1}^{\prime}$ from $w_{d+1}$ to $t_{d+1}^{\prime}$ using $v_{2}$ (Lemma 2.1). In the union of $w_{r} t_{r-1}^{\prime}, t_{r-1}^{\prime} E t_{r}^{\prime}$ and all $(E \cup N)$-bridges of $H$ with all attachments in $t_{r-1}^{\prime} E t_{r}^{\prime} \cup\left\{w_{r}\right\}$, we find a path $R_{r}^{\prime}$ from $x_{p}^{\prime}$ to $t_{r}^{\prime}$ using $w_{r} t_{r-1}^{\prime}$ (Lemma 2.1). In $\left(B+v_{2} v_{1}\right)-\{z\}$ we find a path $R^{\prime}$ from $v_{2}$ to $x_{q}^{\prime}$ using $v_{2} v_{1}$ (Lemma 2.1). Finally modify $t_{d+1}^{\prime} E t_{r-1}^{\prime}$ and $t_{r}^{\prime} E v_{1}$ (both with respect to $\left.\left(H^{\prime}, P^{\prime} \cup Q^{\prime}\right)\right)$ to get two paths which, together with $R_{d+1}^{\prime}, R_{r}^{\prime}-w_{r} t_{r-1}^{\prime}$, $R^{\prime}-v_{2} v_{1}$ and $P^{\prime}$, give $P^{*}$. By the minimality of $B$ (since $\left.x_{q}^{\prime} \neq v_{1}\right), z_{1}$ and $z_{2}$ belong to different $P^{*}$-bridges of $H$ or $\left\{z_{1}, z_{2}\right\} \cap P^{*} \neq \emptyset$. We now extend $P^{*}$ to the desired path $P$ from $x$ to $y$. In $J_{p}$ we find a path $R_{p}$ from $x_{p}^{\prime}$ to $s_{p}$ using $t_{p}$ if $t_{p} \neq t_{q}$ (Lemma 2.1), otherwise not using $t_{p}$ (Lemma 2.5). In $J_{q}$ we find a path $R_{q}$ from $x_{q}^{\prime}$ to $t_{q}$ using $s_{q}$ if $s_{q} \notin R_{p}$ (Lemma 2.1), otherwise not using $s_{q}$ (Lemma 2.5 ). Now we modify $t_{k} C s_{p}$ and $t_{q} C s_{k}$ (both with respect to $\left(G^{\prime}, P^{*} \cup Q^{\prime}\right)$ ) to get two paths which, together with $P^{*}, R_{p}, R_{q}, V$ and $W$, give the desired path $P$.

The following lemma is a direct consequence of Lemma 2.1.

Lemma 3.5. Let $G$ be a 2-connected plane graph with outercycle $C$ and another disjoint facial cycle $D$, and let $x, y \in V(C)$ and $u, v \in V(D)$ be four distinct vertices. Then $G$ has two disjoint paths from $\{x, y\}$ to $\{u, v\}$ such that for every $(P \cup Q)$-bridge $B$ of $G$ we have $|a(B)| \leq 3$.

The next result plays a similar role as Theorem 3.4 in Sections 5 and 6. Its proof is exactly the same as that of Theorem 3.4 except that (in Stages 2 and 3) we use Lemma 3.5 instead of Lemma 3.3.

Theorem 3.6. Let $G$ be a 2-connected plane graph with outercycle $C$ and another facial cycle $D$, and let $x, y \in V(C)$ and $u, v \in V(D)$ be four distinct vertices. Suppose that (1) any simple closed curve in the plane separating $C$ from $D$ intersects $G$ at least 7 times, and (2) any simple curve in the plane from $C$ to $D$ intersects $G$ at least 8 times. Then $G$ has two disjoint paths $P$ and $Q$ from $\{x, y\}$ to $\{u, v\}$ such that for every $(P \cup Q)$-bridge $B$ of $G$, (i) $|a(B)| \leq 4$, and (ii) $|a(B)| \leq 2$ if $(B-a(B)) \cap(C \cup D) \neq \emptyset$.

In order to include the non-orientable surfaces in the rest of this paper, we need a result (similar to Theorem 3.4) for graphs embedded in the projective plane. But first we need the following result from [10].

Lemma 3.7. Let $G$ be a 2-connected graph embedded in the projective plane, and let $e$ be an edge on a homotopically trivial facial cycle $C$ of $G$. Then $G$ has a cycle $T$ using e such that for every $T$-bridge $B$ of $G$, (i) $B$ is planar and $|a(B)| \leq 3$, and (ii) $|a(B)| \leq 2$ if $(B-a(B)) \cap C \neq \emptyset$.

Theorem 3.8. Let $G$ be 2-connected graph embedded in the projective plane $\Sigma$, and let $C$ be a homotopically trivial facial cycle of $G$ with two vertices $x, y \in C$. Suppose that (1) every simple closed curve in $\Sigma$ homotopic to $C$ and disjoint from the open disc bounded by $C$ must intersect $V(G)$ at least 7 times, and (2) every nontrivial simple closed curve intersecting the open disc bounded by $C$ intersects $G$ at least 8 times. Then $G$ has a path $P$ from $x$ to $y$ such that for every $P$-bridge $B$ of $G$, (i) $B$ is planar and $|a(B)| \leq 4$, and (ii) $|a(B)| \leq 2$ if $(B-a(B)) \cap C \neq \emptyset$.

Proof. By condition (2), $G$ contains three homotopically trivial disjoint cycles around $C$. Let $C^{\prime}$ be the cycle in $G$ disjoint from $C$ and homotopic to $C$ such that the subgraph of $G$ contained in the closed annulus bounded by $C$ and $C^{\prime}$ is 
minimal. We define $x_{i}^{\prime}, s_{i}, t_{i}, J_{i}$ exactly the same as in the proof of Theorem 3.4. We also proceed in three stages as in the proof of Theorem 3.4, and we define $E$ and $F$ in the same way (using condition (2)). Now the only difference is that we will use Lemma 3.7 instead of Lemma 3.3. We omit the details.

\section{Planarizing CyCles}

The results of this section are similar to those of Thomassen's in [15]. The objective here is to reduce graphs embedded in a surface to graphs embedded in the sphere. The proofs are essentially the same as those of [15], though we work on general graphs instead of triangulations. But for the sake of completeness we sketch the proofs here.

First we need some terminology about embeddings of graphs in surfaces. We refer to [13] for terms not defined here. By a result of [13], every nonplanar embedding of a graph in a surface (i.e., an embedding in which the graph is not contained in a disc) has a noncontractible cycle. If $C$ is a contractible cycle of $G$ which is embedded in a surface $\Sigma$ other than the sphere, then $C$ bounds a unique open disc which is called the interior of $C$ in $\Sigma$.

Let $G$ be a graph embedded in a surface $\Sigma$. We use $\operatorname{eg}(G, \Sigma)$ to denote the number satisfying $|V(G)|-|E(G)|+|F(G)|=2-e g(G, \Sigma)$, where $F(G)$ is the set of faces. Suppose that $G=\left(G_{1}, \cdots, G_{m}\right)$ is a sequence of graphs and $\Sigma=\left(\Sigma_{1}, \cdots, \Sigma_{m}\right)$ is a sequence of disjoint surfaces such that $G_{i}$ is embedded in $\Sigma_{i}$. Then we also say that $G$ is embedded in $\Sigma$. For technical reason we let $\rho(G, \Sigma)=\infty$ if $\Sigma$ is the sphere. We also write $\operatorname{eg}(G, \Sigma)=\max \left\{\operatorname{eg}\left(G_{i}, \Sigma_{i}\right): i=1, \cdots, m\right\}$ and $\rho(G, \Sigma)=\min \left\{\rho\left(G_{i}, \Sigma_{i}\right): i=1, \cdots, m\right\}$.

If $C=x_{1} \cdots x_{k} x_{1}$ is a cycle in a graph $G$ embedded in a surface $\Sigma$ with eg $(G, \Sigma)=g$, then by cutting $G$ and $\Sigma$ along $C$ we can form a new graph $G^{\prime}$ embedded in a new surface $\Sigma^{\prime}$, or a pair of new graphs $G^{\prime}=\left(G_{1}, G_{2}\right)$ embedded in a pair of new surfaces $\Sigma^{\prime}=\left(\Sigma_{1}, \Sigma_{2}\right)$ (with $G_{i}$ in $\Sigma_{i}$, respectively): if $C$ is orientation preserving, then we first add a cycle $C^{\prime}=x_{1}^{\prime} \cdots x_{k}^{\prime} x_{1}^{\prime}$. If an edge $y x_{i}$ is incident with $C$ on its left side, we delete $y x_{i}$ and add instead $y x_{i}^{\prime}$. We finally attach a disc to $C$ and another disc to $C^{\prime}$ to get the surface $\Sigma^{\prime}$. (Note that we used the same symbol $C$ for cycles in $G$ and $G^{\prime}$.) If $C$ is orientation reversing, we delete $C$ and add a new cycle $C^{\prime}=x_{1} \cdots x_{k} x_{1}^{\prime} \cdots x_{k}^{\prime} x_{1}$ : we have a local orientation at each vertex of $C$ when walking along $C$ in $\Sigma$, and we add the edge $y x_{i}$ if $y x_{i}$ is to the right of $C$ at $x_{i}$ and $y x_{i}^{\prime}$ otherwise. Finally we attach a disc to $C^{\prime}$ to get the surface $\Sigma^{\prime}$. When $C$ is non-contractible, $e g\left(G^{\prime}, \Sigma^{\prime}\right) \leq g-2$ if $C$ is orientation preserving, and $e g\left(G^{\prime}, \Sigma^{\prime}\right)=g-1$ if $C$ is orientation reversing.

Let $C_{1}, \cdots, C_{m}$ be a collection of pairwise disjoint cycles in a connected graph $G$ embedded in a surface $\Sigma$. If cutting along these cycles we obtain a graph $H$ embedded in a disjoint union of spheres, then we say that $\left\{C_{1}, \cdots, C_{m}\right\}$ is a set of planarizing cycles of $G$ in $\Sigma$. We use the following convention throughout this section. Given a graph $G$ in a surface $\Sigma$, we deal with simple curves which intersect $G$ only at its vertices, and the length of a simple curve is the number of vertices in $G$ on this curve.

The following lemma was proved in [15] for triangulations; the proof we present here is essentially the same.

Lemma 4.1. Suppose that $G$ is a connected graph embedded in a surface $\Sigma$ (other than the sphere) with $\rho(G, \Sigma) \geq 2 k+2$ ( $k$ is a positive integer). Then for every 
vertex $x \in V(G)$ and every integer $i$ with $1 \leq i \leq k$ there is an induced contractible cycle $C$ in $G$ containing $x$ in its interior such that for every $z \in V(C)$ there is a simple curve $\gamma$ of length $d_{G \Sigma}(z, x)=i+1$ in the closed disc bounded by $C$ from $z$ to $x$ with $\gamma \cap C=\{z\}$.

Proof. We use induction on $k$. For $k=1, \rho(G, \Sigma) \geq 4$. Let $C$ be a cycle in $G$ which bounds a disc in $\Sigma$ containing $x$ such that for every vertex $z$ on $C$ there is a simple curve in $\Sigma$ from $z$ to $x$ of length 2 in the closed disc bounded by $C$. If $C$ is induced, then $C$ is the desired cycle. Suppose that $C$ has a chord $u v$. Let $P$ and $Q$ be the two paths of $C$ between $u$ and $v$, and let $R$ be a simple curve in the interior of $C$ from $u$ to $v$ meeting $G$ only at $u, v$ and $x$. Then $R \cup P$ and $R \cup Q$ are contractible. Since $\rho(G, \Sigma) \geq 4, R+u v$ is contractible. Hence both $P+u v$ and $Q+u v$ are contractible. We can choose the notation so that $P+u v$ is a contractible cycle containing $x$ and $Q$ in its interior. We replace $C$ by $P+u v$. Continuing this procedure, we eventually obtain the desired cycle.

Hence we may assume that $k \geq 2$. Suppose that we can find desired contractible cycles for $i \leq k-1$. Let $D$ be the contractible cycle with respect to $k-1$. We now contract $D$ and its interior to a single vertex $x^{\prime}$ to obtain a new graph $G^{\prime}$ in $\Sigma$ (loops and multiple edges are deleted). It is easy to check that $\rho\left(G^{\prime}, \Sigma\right) \geq 4$. Thus by the argument in the above paragraph we find an induced contractible cycle $C$ in $G^{\prime}$ around $x^{\prime}$. It is not difficult to check that $C$ is the desired cycle with respect to $i=k$.

Let $C$ be a noncontractible cycle of $G$ which is embedded in a surface $\Sigma$. We cut $G$ and $\Sigma$ along $C$ to get $G^{\prime}$ and $\Sigma^{\prime}$. If $C$ is orientation preserving, then we have two cycles $C$ and $C^{\prime}$ in $G^{\prime}$ (embedded in $\Sigma^{\prime}$ ): in the interior of $C$ and $C^{\prime}$, respectively, we add vertices $x$ and $x^{\prime}$ and join $x$ to each $x_{i}$ and join $x^{\prime}$ to each $x_{i}^{\prime}$. If $C$ is orientation reversing, then we have a single cycle $C^{\prime}$ obtained from $C$ : in this case we simply add a vertex $x^{\prime}$ in the interior of $C^{\prime}$ and join $x^{\prime}$ to each $x_{i}$ and each $x_{i}^{\prime}$. In either case we have a new graph $G^{\prime \prime}$ embedded in $\Sigma^{\prime}$ (or possibly in the orientation preserving case, a pair of new graphs $G^{\prime \prime}=\left(G_{1}^{\prime}, G_{2}^{\prime}\right)$ embedded in $\left.\Sigma^{\prime}=\left(\Sigma_{1}, \Sigma_{2}\right)\right)$.

Lemma 4.2. Let $G$ be a connected graph embedded in a surface $\Sigma$ with $\rho(G, \Sigma) \geq 5$. Then for every shortest nontrivial simple closed curve $\Gamma$ in $\Sigma, G$ has an induced noncontractible cycle $C$ homotopic to $\Gamma$ such that for every vertex $z \in C$, $d_{G \Sigma}(z, \Gamma \cap V(G)) \leq 2$ and after cutting along $C$ we have $\rho\left(G^{\prime \prime}, \Sigma^{\prime}\right) \geq \rho(G, \Sigma) / 2$.

Proof. Let $\Gamma$ be a simple closed curve in $\Sigma$ intersecting $G$ the least number of times. If $\Gamma$ is orientation preserving, then (since $\rho(G, \Sigma) \geq 5$ ) there is a cycle $C$ of $G$ homotopic to $\Gamma$ (we may specify $C$ to be on the left side of $\Gamma$ ) so that for each vertex $z \in C, d_{G \Sigma}(z, \Gamma \cap V(G)) \leq 2$. Suppose that $u v$ is a chord of $C$. Let $P$ and $Q$ be the two subpaths of $C$ between $u$ and $v$. Then one of $P+u v$ and $Q+u v$ must be trivial; otherwise let $P^{\prime}$ (and $Q^{\prime}$ ) be a simple curve along $P$ (and $Q$ ) from $u$ to $v$ which only meet $G$ in $V(G) \cap \Gamma$ such that $P^{\prime} \cup Q^{\prime}$ is a simple closed curve homotopic to $\Gamma$. Let $T$ be a simple curve from $u$ to $v$ along $u v$ intersecting $G$ only at $u$ and $v$. Then $P^{\prime} \cup T$ is homotopic to $P+u v$ and $Q^{\prime} \cup T$ is homotopic to $Q+u v$. Since $\rho(G, \Sigma) \geq 5,\left|P^{\prime} \cap V(G)\right| \geq 5$ and $\left|Q^{\prime} \cap V(G)\right| \geq 5$, and so $P^{\prime}+u v$ is a nontrivial simple closed curve shorter than $\Gamma$, a contradiction. Hence let $P+u v$ be trivial. We replace $C$ by $Q+u v$ (which is homotopic to $\Gamma$ ). Continuing this procedure, we obtain the desired induced cycle $C$. The case that $\Gamma$ is orientation reversing can 
be treated in the same way (where we use the local orientation at each vertex of $C$ when we find $P^{\prime}$ and $\left.Q^{\prime}\right)$.

We now cut $G$ and $\Sigma$ along $C$ and obtain $G^{\prime \prime}$ in $\Sigma^{\prime}$. We prove that $\rho\left(G^{\prime \prime}, \Sigma^{\prime}\right) \geq$ $\rho(G, \Sigma) / 2$. If $\Sigma^{\prime}$ is the sphere, then $\rho\left(G^{\prime \prime}, \Sigma^{\prime}\right)=\infty>\rho(G, \Sigma) / 2$. So we may assume that $\Sigma^{\prime}$ is not the sphere. Let $\gamma$ be a nontrivial simple closed curve in $\Sigma^{\prime}$.

Suppose first that $C$ is orientation preserving. If $\gamma$ contains a simple curve $\gamma^{\prime}$ from $C$ to $C^{\prime}$ meeting $C \cup C^{\prime}$ only at its ends, then in $\Sigma$, $\gamma^{\prime}$ starts from the left side of $C$ and ends on the right side of $C$. We can extend $\gamma^{\prime}$ slightly if necessary that $\gamma^{\prime}$ is from the left side of $\Gamma$ to the right side of $\Gamma$, and let $P$ be the shorter subcurve of $\Gamma$ between the two ends of $\gamma^{\prime}$. Then $P \cup \gamma^{\prime}$ is a nontrivial simple closed curve in $\Sigma$, and so must intersect $G$ at least $\rho(G, \Sigma)$ times. Hence $\gamma^{\prime}$ ( and also $\gamma$ ) intersects $G^{\prime \prime}$ at least $\rho(G, \Sigma) / 2$ times. Therefore we may assume that no subcurve of $\gamma$ goes from $C$ to $C^{\prime}$ in $\Sigma^{\prime}$. If $\gamma$ does not go through $x$ or $x^{\prime}$, then $\gamma$ is a nontrivial simple closed curve in $\Sigma$, and so intersects $G^{\prime \prime}$ at least $\rho(G, \Sigma)$ times. Thus we may assume that $\gamma$ goes through $x^{\prime}$ but not $x$ (or equivalently through $x$ but not $x^{\prime}$ ). Then $\gamma$ has a subcurve $R$ joining two vertices $y$ and $z$ on $C^{\prime}$ with $R \cap C^{\prime}=\{y, z\}$ such that $R$ together with a simple curve from $y$ to $z$ intersecting $G^{\prime \prime}$ only at $y, z, x^{\prime}$ is nontrivial. We may extend $R$ to meet $\Gamma$, and let $P$ be the shorter subcurve of $\Gamma$ between the two ends of $R$. Then $P \cup R$ is also nontrivial in $\Sigma$. Hence $R$ (and also $\gamma$ ) intersects $G$ at least $\rho(G, \Sigma) / 2$ times.

Now let $C$ be orientation reversing and let $C^{\prime}=x_{1} \cdots x_{k} x_{1}^{\prime} \cdots x_{k}^{\prime} x_{1}$. If $x^{\prime} \notin \gamma$ and $\gamma$ meets $C^{\prime}$ at most once, then $\gamma$ is also a nontrivial simple closed curve in $\Sigma$, and so $\gamma$ intersects $G^{\prime \prime}$ at least $\rho(G, \Sigma)$ times. So we may assume that $\gamma$ intersects $C^{\prime}$ at least twice. Hence $\gamma$ has a subcurve $P$ intersecting $C^{\prime}$ only at its ends, say $y$ and $z$, such that $P+y x^{\prime} z$ is nontrivial in $\Sigma^{\prime}$. If $\{y, z\}=\left\{x_{i}, x_{i}^{\prime}\right\}$, then $P$ is a nontrivial simple closed curve in $\Sigma$, and so $\gamma$ intersects $G^{\prime \prime}$ at least $\rho(G, \Sigma) / 2$ times. Hence we may assume that $\{y, z\} \neq\left\{x_{i}, x_{i}^{\prime}\right\}$. We may extend $P$ to meet $\Gamma$, and let $Q$ and $R$ be the two subcurves of $\Gamma$ between the two ends of $P$. Both $P \cup Q$ and $P \cup R$ are nontrivial in $\Sigma$; for suppose that $P \cup Q$ is trivial in $\Sigma$, then it bounds a disc in $\Sigma$ which implies that $P \cup Q$ bounds a disc in $\Sigma^{\prime}$, contradicting the fact that $P \cup Q$ is homotopic to $P+y x^{\prime} z$ in $\Sigma^{\prime}$. Hence $\gamma$ must have length at least $\rho(G, \Sigma) / 2$.

Note that in the above lemma $\rho(G, \Sigma) \geq 3$ is sufficient if we do not ask $C$ to be induced. We now reduce graphs embedded in a surface other than the sphere to a graph embedded in a disjoint union of spheres.

Theorem 4.3. Let $G$ be a connected graph embedded in a surface $\Sigma$ (other than the sphere) with $\mathrm{eg}(G, \Sigma)=g$ and $\rho(G, \Sigma) \geq 8(d+1)\left(2^{g}-1\right)$. Then $G$ can be reduced to a graph $H$ embedded in a disjoint union $S$ of spheres by cutting along a set of planarizing cycles $\left\{C_{1}, \cdots, C_{m}\right\}$ (in this order) such that

(i) each $C_{i}$ is induced,

(ii) for every integer $k$ with $0 \leq k \leq d / 2$ there is an induced cycle $D_{i}^{\prime k}$ (and $D_{i}^{k}$ if $C_{i}$ is orientation preserving) which bounds a closed disc in $S$ containing $C_{i}^{\prime}$ (and $C_{i}$ ) such that for every vertex $z \in D_{i}^{\prime k}$ (and $z \in D_{i}^{k}$ ) there is a simple curve $P$ in $S$ from $z$ to $C_{i}^{\prime}\left(\right.$ and $\left.C_{i}\right)$ with length equal to $d_{H S}\left(z, C_{i}^{\prime}\right)=k+1\left(\right.$ and $\left.d_{H S}\left(z, C_{i}\right)=k+1\right)$ and $P \cap D_{i}^{\prime k}=\{z\}\left(\right.$ and $\left.P \cap D_{i}^{k}=\{z\}\right)$, and

(iii) all $D_{i}^{k}$ and $D_{i}^{\prime k}$ are disjoint, and for each integer $k$ with $0 \leq k \leq d / 2$, the closed disc bounded by $D_{i}^{\prime k}$ containing $C_{i}^{\prime}$ is disjoint from the closed disc bounded 
by $D_{i}^{k}$ containing $C_{i}$, and both do not contain the disc bounded by $D_{j}^{\prime k}$ or $D_{j}^{k}$ containing $C_{j}^{\prime}$ or $C_{j}$ for any $j>i$.

Proof. We use induction on $e g(G, \Sigma)=g$. By Lemma 4.2, given a shortest nontrivial simple closed curve $\Gamma$ in $\Sigma$, we find an induced noncontractible cycle $C$ in $G$ homotopic to $\Gamma$ such that after cutting along $C$ we have $\rho\left(G^{\prime \prime}, \Sigma^{\prime}\right) \geq \rho(G, \Sigma) / 2$ (also for every vertex $z$ on $C, d_{G \Sigma}(z, \Gamma \cap V(G)) \leq 2$ ).

First, we deal with the case that $\Sigma^{\prime}$ is the sphere. If $C$ is orientation preserving, then let $C_{1}=C$. By a similar argument as in the proof of Lemma 4.2, $d_{G^{\prime} \Sigma^{\prime}}\left(C, C^{\prime}\right) \geq \rho(G, \Sigma) / 2 \geq 4(d+1)$. Let $D_{1}^{0}=C$ and $D_{1}^{\prime 0}=C_{1}^{\prime}$. Suppose we have constructed $D_{1}^{k}$ for $0 \leq k \leq d / 2-1$. Let $F^{k}$ be the facial cycle of the block of $G^{\prime}-D_{1}^{k}$ containing $C^{\prime}$ which is not a facial cycle of $G^{\prime}$. If $F^{k}$ is induced, then let $D_{1}^{k+1}=F^{k}$. Otherwsie let $u v$ be a chord of $F^{k}$. Now one of the two cycles in $F^{k}+u v$ containing $u v$ separates $C$ from $C^{\prime}$, and we use such a cycle to replace $F^{k}$. Continuing this procedure we eventually get an induced cycle $D_{1}^{k+1}$. Similarly we can construct $D_{1}^{\prime k}$ for $0 \leq k \leq d / 2$. Note that conditions (i) and (ii) are satisfied because of the construction, and condition (iii) is satisfied since $d_{G^{\prime} \Sigma^{\prime}}\left(C, C^{\prime}\right) \geq 4(d+1)$.

Now assume $C$ is orientation reversing. In this case $\Sigma$ is the projective plane. Let $x \in V(G)$. We first find a noncontractible cycle $C_{1}$ such that $d_{G \Sigma}\left(x, C_{1}\right) \geq 4 d$. By Lemma 4.1, there are disjoint contractible cycles $F_{i}$ for $1 \leq i \leq 4 d+3$ such that $F_{i}$ contains $x, F_{1}, \cdots, F_{i-1}$ in its interior for $i \geq 2$. If $C$ does not intersect the open disc bounded by $F_{4 d+3}$, then $C_{1}=C$. Otherwise, $C$ contains a subpath $P$ from $x$ to $y$, where $x, y \in F_{4 d+3}$, such that $P$ and a subpath $Q$ of $F_{4 d+3}$ between $x$ and $y$ form a noncontractible cycle $C_{1}$. Clearly $C_{1}$ does not intersect the open disc bounded by $F_{4 d+3}$. All possible chords of $C_{1}$ are from $P$ to $Q$, and so we may assume that $C_{1}$ is induced (or else we use the same argument as in proof of Lemma 4.1 to get rid of those chords). Clearly $d_{G \Sigma}\left(x, C_{1}\right) \geq 4 d$. Now after cutting along $C_{1}$ we obtain a graph $G^{\prime}$ embedded in the sphere $\Sigma^{\prime}$ with a cycle $C_{1}^{\prime}$ and $d_{G^{\prime} \Sigma^{\prime}}\left(x, C_{1}^{\prime}\right) \geq 4 d$. Let $D_{1}^{\prime 0}=C_{1}^{\prime}$. Suppose we have constructed $D_{1}^{\prime k}$ for $0 \leq k \leq d / 2-1$. Let $F^{\prime k}$ be the facial cycle of the block of $G^{\prime}-D_{1}^{\prime k}$ containing $x$ which is not a facial cycle of $G^{\prime}$. Let $D_{1}^{\prime k+1}=F^{\prime k}$ if $F^{\prime k}$ is induced. Otherwise let $u v$ be a chord of $F^{\prime k}$. Then one of the cycles in $F^{\prime k}+u v$ containing $u v$ separates $C_{1}^{\prime}$ from $x$, and we use such a cycle to replace $F^{\prime k}$. Continuing this procedure we will eventually get the desired induced cyle $D_{1}^{\prime k+1}$. Clearly conditions (i)-(iii) are satisfied.

We now assume that after cutting along $C, G^{\prime}$ is embedded in $\Sigma^{\prime}$ other than the sphere. By Lemma $4.2, \rho\left(G^{\prime \prime}, \Sigma^{\prime}\right) \geq 4(d+1)\left(2^{g}-1\right)$. By Lemma 4.1 we have the contractible induced cycles $D^{\prime k}$ (and $D^{k}$ if $C$ is orientation preserving) in $\Sigma^{\prime}$ such that for every vertex $z \in D^{\prime k} \cup D^{k}$ there is a simple curve from $z$ to $x^{\prime}$ (and to $x$ ) in the closed disc in $\Sigma^{\prime}$ bounded by $D^{\prime k}$ (and $D^{k}$ ) of length $d_{G^{\prime} \Sigma^{\prime}}\left(z, x^{\prime}\right)=k+1$ (and $\left.d_{G^{\prime} \Sigma^{\prime}}(z, x)=k+1\right)$ meeting $D^{\prime k} \cup D^{k}$ exactly at $z$, where $D^{\prime 0}=C^{\prime}$ (and $D^{0}=C$ ) and $k \leq \rho\left(G^{\prime \prime}, \Sigma^{\prime}\right) / 2-2$.

We claim that when $C$ is orientation preserving the closed disc in $\Sigma^{\prime}$ bounded by $D^{\prime d+1}$ containing $C^{\prime}$ is disjoint from the closed disc in $\Sigma^{\prime}$ bounded by $D^{d+1}$ containing $C$. First we show that in $\Sigma^{\prime}$ the closed disc bounded by $D^{\prime d+1}$ containing $C^{\prime}$ does not contain $C$. Otherwise let $C$ be contained in the interior of $D^{\prime k}$ with $k$ minimum $(k \leq d+1)$. Then there is a simple curve $P$ of length at most $2 k+2$ in the interior of $D^{\prime k}$ (in $\Sigma^{\prime}$ ) meeting $C^{\prime}$ only at its ends such that the two new closed curves in $P \cup C^{\prime}$ contain $C$ in their interiors (in $\Sigma^{\prime}$ ). We may extend $P$ a little to 
meet $\Gamma$. Let $Q$ and $R$ be the two subcurves of $\Gamma$ between the two ends of $P$. Then both $P \cup Q$ and $P \cup R$ are nontrivial in $\Sigma$. Since $\Gamma$ is shortest in $\Sigma, \Gamma$ would have length at most $2(2 k+4)-2 \leq 4 d+6$, a contradiction. Similarly we can prove that the closed disc in $\Sigma^{\prime}$ bounded by $D^{d+1}$ containing $C$ does not contain $C^{\prime}$. Hence if the closed disc in $\Sigma^{\prime}$ bounded by $D^{\prime d+1}$ containing $C^{\prime}$ intersects the closed disc bounded by $D^{d+1}$ containing $C$, then in $\Sigma$ there is a simple curve from the left side of $C$ to the right side of $C$ of length at most $2 d+3$. But this would imply that $\Gamma$ has length at most $4 d+6$, a contradiction.

Now let $E^{\prime}=D^{\prime d+1}$ (and let $E=D^{d+1}$ if $C$ is orientation preserving). We form a new graph $G_{0}$ embedded in $\Sigma^{\prime}$ by contracting the interior of $E^{\prime}$ (and the interior of $E$ in the orientation preserving case) to a vertex $y^{\prime}$ (and $y$ ) and deleting the resulting loops and multiple edges.

We now verify that $\rho\left(G_{0}, \Sigma^{\prime}\right) \geq \rho\left(G^{\prime \prime}, \Sigma^{\prime}\right)-4(d+1)$. Let $\gamma$ be a nontrivial simple closed curve in $\Sigma^{\prime}$. If $\gamma$ does not contain any of $y$ or $y^{\prime}$, then $\gamma$ intersects $V\left(G_{0}\right)$ only at vertices of $V\left(G^{\prime \prime}\right)$, and so $\gamma$ has length at least $\rho\left(G^{\prime \prime}, \Sigma^{\prime}\right)$. If $\gamma$ uses $y^{\prime}$, then $\gamma$ has a subcurve from $z_{1} \in E^{\prime}$ to $z_{2} \in E^{\prime}$ which only intersects $V\left(G_{0}\right)$ at $y^{\prime}, z_{1}$ and $z_{2}$. We can replace this subcurve of $\gamma$ by a simple curve in $\Sigma^{\prime}$ from $z_{1}$ to $z_{2}$ in the interior of $E^{\prime}$ (in $G^{\prime \prime}$ ) which intersects $V\left(G^{\prime \prime}\right)$ at most $2(d+1)+1$ times. A similar modification is performed if $\gamma$ contains $y$. Thus $\gamma$ intersects $V\left(G_{0}\right)$ at least $\rho\left(G^{\prime \prime}, \Sigma^{\prime}\right)-4(d+1)$ times. Hence $\rho\left(G_{0}, \Sigma^{\prime}\right) \geq 8(d+1)\left(2^{g-1}-1\right)$.

We apply induction to $G_{0}$ which is embedded in the surface $\Sigma^{\prime}$. We find a set of induced disjoint cycles $\left\{C_{2}, \cdots, C_{m}\right\}$ and by cutting along these cycles in the order $C_{2}, \cdots, C_{m}$ we reduce $G_{0}$ to a graph $H_{0}$ in a disjoint union $S$ of spheres. We now obtain a graph $H$ from $H_{0}$ by replacing $y^{\prime}$ and $y$ by the interiors of $D^{\prime d+1}$ and $D^{d+1}$ in $G^{\prime}$ (in $\Sigma^{\prime}$ ). We also modify the cycles $\left\{C_{2}, \cdots, C_{m}\right\}$ to get the desired set of planarizing cycles in $G$. If $y$ is used by some $C_{j}(2 \leq j \leq m)$, then let uyv be a subpath of $C_{j}$, where $u, v \in V\left(C_{j}\right)$. Since $u y, v y \in E\left(G_{0}\right)$, there are disjoint paths from $u$ and $v$ to the interior of $E$ (in $\Sigma^{\prime}$ ) which are internally disjoint from $E$. We can rewrite $C_{j}$ by replacing $u y v$ by the union of a path from $u$ to $y_{1}, y_{1} \cdots y_{r}$, and a path from $v$ to $y_{r}$, where $y_{1} \cdots y_{r} \subset G^{\prime}$ is in the interior of $E$ in $\Sigma^{\prime}$ and $d_{G^{\prime} \Sigma^{\prime}}\left(y_{i}, E\right)=1$ for each $i$. We do the same when $y^{\prime}$ is used by $C_{j}$. Clearly $H$ and $\left\{C_{1}, \cdots, C_{m}\right\}$ are as desired.

\section{HAMilton CYCles}

We now prove the following result, establishing a conjecture of Thomassen [14].

Theorem 5.1. Let $G$ be a 5-connected triangulation of a surface $\Sigma$ with eg $(G, \Sigma)=$ $g$ and $\rho(G, \Sigma) \geq 96\left(2^{g}-1\right)$. Then $G$ has a Hamilton cycle.

Proof. By Theorem 4.3 we can cut the surface along a set of planarizing cycles to get a new graph $H$ embedded in a disjoint union of spheres with disjoint induced cycles $C_{i}, C_{i}^{\prime}, D_{i}^{5}$, and $D_{i}^{\prime 5}$ for $i=1, \cdots, m$. Note that $d=11$. Since $G$ is a triangulation, all faces of $H$ are triangles except possibly those bounded by $C_{i}$ or $C_{i}^{\prime}, i=1, \cdots, m$.

When $C_{i}$ is orientation preserving, let $H_{i}$ be the graph obtained from $H$ by taking the union of the subgraph of $H$ contained in the closed disc bounded by $D_{i}^{\prime 5}$ containing $C_{i}^{\prime}$ and the subgraph of $G$ contained in the closed disc bounded by $D_{i}^{5}$ containing $C_{i}$ (note that these two subgraphs of $H$ are disjoint), and identifying $C_{i}$ and $C_{i}^{\prime}$ back to $C_{i}$. We view $H_{i}$ as a plane graph with outercycle $D_{i}^{\prime 5}$ and another disjoint facial cycle $D_{i}^{5}$. Note that every simple curve in the plane from $D_{i}^{5}$ to 
$D_{i}^{\prime 5}$ must intersect $H_{i}$ at least 11 times. Let $M_{i}$ be the block of $H_{i}-\left(D_{i}^{5} \cup D_{i}^{\prime 5}\right)$ containing the cycle $C_{i}=C_{i}^{\prime}$, and $F_{i}$ and $F_{i}^{\prime}$ be the new facial cycles of $M_{i}$ with $F_{i}^{\prime}$ being the outercycle.

When $C_{i}$ is orientation reversing, let $H_{i}$ be the subgraph of $H$ contained in the closed disc bounded by $D_{i}^{\prime 5}$ containing $C_{i}^{\prime}$, and then identify $C_{i}^{\prime}$ back to $C_{i}$. We view $H_{i}$ as a graph embedded in the projective plane with a facial cycle $D_{i}^{\prime 5}$. Let $M_{i}$ be the block of $H_{i}-D_{i}^{\prime 5}$ containing $C_{i}$, and let $F_{i}^{\prime}$ be the facial cycle of $M_{i}$ such that every $\left(M_{i} \cup D_{i}^{\prime 5}\right)$-bridge of $H_{i}$ has at most one attachment on $F_{i}^{\prime}$.

Since for every $z \in D_{i}^{5} \cup D_{i}^{\prime 5}$ there is a simple curve in $\Sigma$ from $z$ to $C_{i}$ or $C_{i}^{\prime}$ intersecting $G$ exactly 6 times, every $\left(M_{i} \cup D_{i}^{\prime 5} \cup D_{i}^{5}\right)$-bridge has either one attachment on $D_{i}^{\prime 5} \cup D_{i}^{5}$ or two attachments on $D_{i}^{\prime 5}$ or $D_{i}^{5}$ which are adjacent vertices.

Claim 1. Every $\left(M_{i} \cup D_{i}^{5} \cup D_{i}^{\prime 5}\right)$-bridge of $H_{i}$ is an edge.

Proof of Claim 1. Suppose that $B$ is a $\left(M_{i} \cup D_{i}^{5} \cup D_{i}^{\prime 5}\right)$-bridge of $H_{i}$ which is not an edge. Since $G$ is 5-connected, $B$ must contain exactly one of $C_{i}^{\prime}$ or $C_{i}$. But this implies that there is a nontrivial simple closed curve in $\Sigma$ (along $B$ ) intersecting $G$ only at $a(B)$ (which has at most three vertices), a contradiction.

For $i=1,2, \cdots, m$ and in this order, we replace with a vertex $u_{i}^{\prime}$ (or a vertex $u_{i}$ ) the edges and vertices contained in the open disc bounded by $D_{i}^{\prime 5}$ (or bounded by $D_{i}^{5}$ ) containing $C_{i}^{\prime}$ (or $C_{i}$ ), and then add edges from $u_{i}^{\prime}$ (or $u_{i}$ ) to every vertex on $D_{i}^{\prime 5}$ (or $D_{i}^{5}$ ) which is adjacent to at least two vertices on $F_{i}^{\prime}$ (or $F_{i}$ ). We use $H^{\prime}$ to denote the resulting graph. Note that $H^{\prime}$ needs not to be connected. Similarly we can obtain a graph $M_{i}^{\prime}$ from $M_{i}$ by replacing by $u_{j}^{\prime}$ or $u_{j}$ those open discs bounded $D_{j}^{\prime 5}$ or $D_{j}^{5}$ containing $C_{j}^{\prime}$ or $C_{j}$ which are contained in $M_{i}$, where $M_{i}^{\prime}=M_{i}$ if $M_{i}$ contains no other $C_{j}^{\prime}$ or $C_{j}$.

Note that the degrees of $u_{i}^{\prime}$ and $u_{i}$ in $H^{\prime}$ or $M_{i}^{\prime}$ are sufficiently large (by the large representativity and the fact that $G$ is a triangulation).

Claim 2. Every component of $H^{\prime}$ is 4 -connected.

Proof of Claim 2. Suppose that a component of $H^{\prime}$ has a cutset $S$ of size less than or equal to 3. By the choice of $D_{i}^{5}$ and $D_{i}^{\prime 5}$, we may assume that $\left|S \cap D_{i}^{\prime 5}\right| \geq 2$ (or equivalently, $\left.\left|S \cap D_{i}^{5}\right| \geq 2\right)$. Note that $G$ is a triangulation. If $u_{i}^{\prime} \in S$, then $D_{i}^{\prime 5}$ has a chord, a contradiction. So $u_{i}^{\prime} \notin S$. Let $A$ be the component of $H^{\prime}-S$ not containing $u_{i}^{\prime}$. Then all vertices in $\left(A \cap D_{i}^{\prime 5}\right)-S$ are adjacent to exactly one vertex of $F_{i}^{\prime}$, say $v$, and so $S \cup\{v\}$ is a 4-cut of $G$, a contradiction. Thus each component of $H^{\prime}$ is 4 -connected.

By Claim 2, each component of $H^{\prime}$ has a Hamilton cycle. We use $T$ to denote the union of all those Hamilton cycles. Let the two vertices on $T$ adjacent to $u_{i}^{\prime}$ (or $u_{i}$ ) be $p_{i}^{\prime}$ and $q_{i}^{\prime}$ (or $p_{i}$ and $q_{i}$ ). By the choice of $u_{i}^{\prime}$ and $u_{i}$, we may assume that $p_{i}^{\prime}$ and $q_{i}^{\prime}$ are adjacent to $x_{i}^{\prime}$ and $y_{i}^{\prime}$ in $F_{i}^{\prime}$, respectively, and that $p_{i}$ and $q_{i}$ are adjacent to $x_{i}$ and $y_{i}$ in $F_{i}$, respectively. Note that we may choose $x_{i}^{\prime} \neq y_{i}^{\prime}$ and $x_{i} \neq y_{i}$.

Claim 3. Every 3-cut of $M_{i}^{\prime}$ must have two vertices on $F_{i}^{\prime}$ or on $F_{i}$ and each $M_{i}^{\prime}$ is 3-connected.

Proof of Claim 3. The first half of the claim follows from the same argument as in the proof of the above claim. 
Suppose that $S$ is a 2-cut of $M_{i}^{\prime}$. We may assume that $S \subset F_{i}^{\prime}$ (or equivalently, $S \subset F_{i}$ ). Let $A$ be the component of $M_{i}^{\prime}-S$ not containing $C_{i}$. Since $D_{i}^{\prime 4}$ is induced and $G$ is a triangulation, $S$ cannot be contained in $D_{i}^{\prime 4}$. Since every vertex of $D_{i}^{\prime 5}$ has a simple curve in $\Sigma$ to $C_{i}$ intersecting $G$ exactly 5 times, $A$ is incident to at most two vertices on $D_{i}^{\prime 5}$ which must be adjacent. Hence $S$ together with the vertices of $D_{i}^{\prime 5}$ incident with $A$ forms a 4-cut, a contradiction. Therefore $M_{i}^{\prime}$ is 3 -connected.

We now extend these disjoint paths $T-\left\{u_{i}, u_{i}^{\prime}: i=1, \cdots, m\right\}$ to a Hamilton cycle of $G$ by including those vertices in $M_{i}^{\prime}$.

If $C_{m}$ is orientation reversing, then we just use Theorem 3.8 to find a path $P_{m}^{\prime}$ in $M_{m}^{\prime}$ from $x_{m}^{\prime}$ to $y_{m}^{\prime}$. Let $T_{1}=\left(T-u_{m}^{\prime}\right) \cup P_{m}^{\prime} \cup\left\{p_{m}^{\prime} x_{m}^{\prime}, q_{m}^{\prime} y_{m}^{\prime}\right\}$. If $C_{m}$ is orientation preserving and $u_{m}$ and $u_{m}^{\prime}$ are in the same component of $T$, then we use Theorem 3.4 to find two disjoint paths $P_{m}$ and $P_{m}^{\prime}$ with $P_{m}$ from $x_{m}$ to $y_{m}$ and $P_{m}^{\prime}$ from $x_{m}^{\prime}$ to $y_{m}^{\prime}$; otherwise we use Theorem 3.6 to find two disjoint paths $P_{m}$ and $P_{m}^{\prime}$ from $\left\{x_{m}, y_{m}\right\}$ to $\left\{x_{m}^{\prime}, y_{m}^{\prime}\right\}$. Let $T_{1}=\left(T-\left\{u_{m}, u_{m}^{\prime}\right\}\right) \cup P_{m} \cup P_{m}^{\prime} \cup$ $\left\{p_{m} x_{m}, q_{m} y_{m}, p_{m}^{\prime} x_{m}^{\prime}, q_{m}^{\prime} y_{m}^{\prime}\right\}$. By Claim $3, T_{1}$ uses all vertices in $M_{m}^{\prime}$. Suppose that we have included vertices in $M_{j}^{\prime}$ and the current $T_{m-j+1}$ uses all vertices in $M_{j}^{\prime}$. For $u_{i}^{\prime}, u_{i} \in M_{j}^{\prime}$, we define $x_{i}, x_{i}^{\prime}, y_{i}, y_{i}^{\prime}, p_{i}, q_{i}, p_{i}^{\prime}, q_{i}^{\prime}$ much the same as we did for those from $T$. We then in $M_{j-1}^{\prime}$ find two disjoint paths $P_{j-1}$ and $P_{j-1}^{\prime}$ (or a path $P_{j-1}^{\prime}$ when $C_{j}$ is orientation reversing) by Theorem 3.4 or Theorem 3.6 (or Theorem 3.8). By the above claim $T_{m-j+2}$ uses all vertices of $M_{j-1}^{\prime}$.

Note that conclusion (iii) of Theorem 4.3 guarantees that we can safely continue this procedure to find paths $P_{i}^{\prime}$ and/or $P_{i}$ in $M_{i}^{\prime}$. It is now easy to see that $T_{m}$ is a Hamilton cycle of $G$.

To end this section, we point out that our technique fails for the general 5connected graphs because we cannot find a way to get $M_{i}^{\prime} 3$-connected, where the main problem is that a 2 -cut may be contained in $D_{i}^{\prime 4}$. It would be interesting to know if 5-connected graphs with sufficiently large representativity are hamiltonian.

\section{2-WALKS AND 3-WALKS}

We end this paper by presenting an application of the generalized version of Thomassen's planarizing cycles. A chain of blocks is either a 2-connected graph or a connected graph in which every block contains at most two cutvertices and every cutvertex is contained in exactly two blocks. In a chain of blocks an endblock is a block containing at most one cutvertex. A circuit graph $(G, C)$ is a 2-connected plane graph $G$ with a facial cycle $C$ such that for any 2-cut $T$ of $G$, each $T$-bridge of $G$ must contain a vertex of $C$. An annulus graph $\left(G, C_{1}, C_{2}\right)$ is a 2-connected plane graph with two facial cycles $C_{1}$ and $C_{2}$ such that for each 2-cut $T$ of $G$, every $T$-bridge of $G$ contains a vertex of $C_{1} \cup C_{2}$.

The following result is from [5] which was used by Gao and Richter to establish a conjecture of Jackson and Wormald.

Lemma 6.1. Let $(G, C)$ be a circuit graph and $x, y \in V(C)$. Then $G$ has a 2-walk that visits each of $x$ and $y$ only once and traverses every edge of $C$ exactly once.

We need the following easy generalization of Lemma 6.1.

Lemma 6.2. Let $G$ be a plane graph which is a chain of blocks $G_{1}, \cdots, G_{n}$ such that $\left(G_{i}, C_{i}\right)$ is a circuit graph, where $C_{i}$ is the outercycle of some $G_{i}$. Let $x \in V\left(C_{1}\right)$ 
and $y \in V\left(C_{n}\right)$ such that neither $x$ nor $y$ is a cutvertex of $G$. Then $G$ has a 2-walk which visits $x$ and $y$ exactly once.

It is not difficult to see that if a graph has a 2-walk, then it also has a spanning tree with maximum degree at most 3 . Thomassen [14] showed that every triangulation of orientable genus $g$ with no noncontractible cycle of length less than $2^{3 g+4}$ contains a spanning tree of maximum degree at most 4. He also conjectured in [14] that the maximum degree can be lowered to 3 provided that the triangulation is 4-connected. This was verified by Ellingham and Gao [4] and they need $2^{3 g+5}$ for the length of a shortest noncontractible cycle. In this section we prove the following stronger result.

Theorem 6.3. Let $G$ be a 4-connected graph embedded in a surface $\Sigma$ with eg $(G, \Sigma)$ $=g$ and $\rho(G, \Sigma) \geq 48\left(2^{g}-1\right)$. Then $G$ has a 2 -walk.

Proof. We use Theorem 4.3 and an idea similar to that in the proof of Theorem 5.1. We know that $G$ has a set of planarizing cycles $C_{1}, \cdots, C_{m}$ with $D_{i}^{2}$ and $D_{i}^{\prime 2}$ as in Theorem 4.3. Note that $d=5$. We now cut $G$ along these cycles to obtain a graph $H$ (embedded in a disjoint union of spheres) with disjoint induced cycles $C_{i}^{\prime}$ and $D_{i}^{\prime 2}$ (and $C_{i}$ and $D_{i}^{2}$ if $C_{i}$ is orientation preserving). Let $H_{i}, M_{i}, M_{i}^{\prime}, F_{i}^{\prime}$ and $F_{i}$ be defined the same as in the proof of Theorem 5.1 (note that we use $D_{i}^{\prime 2}$ and $D_{i}^{2}$ instead of $D_{i}^{\prime 5}$ and $D_{i}^{5}$ ). Unlike the case in Theorem 5.1, $M_{i}^{\prime}$ may have 2-cuts contained in $F_{i}^{\prime}$ or in $F_{i}$.

As in the proof of Theorem 5.1, every $\left(M_{i} \cup D_{i}^{2} \cup D_{i}^{\prime 2}\right)$-bridge of $H_{i}$ is an edge. By a result on matchings in bipartite graphs (see [1] for example) and by the fact that $G$ has very large representativity, we conclude that there is a very large matching $A_{i}^{\prime}$ (or $A_{i}$ ) between $D_{i}^{\prime 2}$ and $F_{i}^{\prime}$ (or between $D_{i}^{2}$ and $F_{i}$ ).

We now construct new graphs $H^{\prime}$ and $M_{i}^{\prime}$ the same as in the proof of Theorem 5.1 , and we use the same notation for vertices $u_{i}^{\prime}$ and $u_{i}$, where we join $u_{i}^{\prime}$ (and/or $u_{i}$ ) to the vertices in $D_{i}^{\prime 2} \cap V\left(A_{i}^{\prime}\right)$ (and/or $D_{i}^{2} \cap V\left(A_{i}\right)$ ).

As in the proof of Theorem 5.1, we find a cycle $T_{m}$ in $G$ by finding disjoint cycles $T=T_{0}$ in $H^{\prime}$ using Lemma 2.1, and then finding paths $P_{i}^{\prime}$ and $P_{i}$ in each $M_{i}^{\prime}$ using Lemma 3.3 or Lemma 3.7 (or Lemma 3.5 if $u_{i}$ and $u_{i}^{\prime}$ are in different components of $\left.T_{m-i}\right)$. This can be done as every $u_{i}, u_{i}^{\prime} \in H^{\prime}$ is used by $T$ and every $u_{i}, u_{i}^{\prime} \in M_{j}^{\prime}$ is used by $P_{j}^{\prime} \cup P_{j}$. Clearly every $T_{m}$-bridge of $G$ is either a $T$-bridge of $H^{\prime}$ containing a vertex of $D_{i}^{2} \cup D_{i}^{\prime 2}$ not as an attachment or a $\left(P_{i}^{\prime} \cup P_{i}\right)$-bridge of $M_{i}^{\prime}$ (or a $P_{i}^{\prime}$-bridge of $M_{i}^{\prime}$ ) containing a vertex of $F_{i}^{\prime} \cup F_{i}$ (or $F_{i}^{\prime}$ ) not as an attachment. We now extend $T_{m}$ to a 2-walk of $H$ by including vertices in those $T$-bridges of $H^{\prime}$ and those $P_{i}^{\prime}$-bridges or $\left(P_{i} \cup P_{i}^{\prime}\right)$-bridges of $M_{i}^{\prime}$.

First we include those vertices in $T$-bridges of $H^{\prime}$ containing a vertex of $D_{i}^{\prime 2} \cup D_{i}^{2}$ not as an attachment. Without loss of generality we may assume that $B_{1}, \cdots, B_{k}$ are those $T$-bridges of $H^{\prime}$ appearing on $D_{i}^{\prime 2}$ in this order. Then by symmetry we may assume that $a\left(B_{j}\right)=\left\{a_{j}, b_{j}, c_{j}\right\}$, where $a_{j} D_{i}^{\prime 2} b_{j} \subset B_{j}$. Since $G$ is 4-connected, $B_{j}-\left\{b_{j}, c_{j}\right\}$ is a chain of blocks with $a_{j}$ in an endblock not as a cutvertex. By Lemma 6.2 we can find a 2 -walk $W_{j}$ in $B_{j}-\left\{b_{j}, c_{j}\right\}$ such that $a_{j}$ is only visited once by $W_{j}$ and we attach $W_{j}$ to $T_{m}$. Similarly, we can include all vertices of $T$-bridges of $H^{\prime}$ containing a vertex of $D_{i}^{2}$.

Now we consider those vertices in $\left(P_{i} \cup P_{i}^{\prime}\right)$-bridges or $P_{i}^{\prime}$-bridges of $M_{i}^{\prime}$. Let $B_{1}^{\prime}, \cdots, B_{n}^{\prime}$ be the $\left(P_{i} \cup P_{i}^{\prime}\right)$-bridges of $M_{i}^{\prime}$ on $F_{i}^{\prime}$ in this order. Let $a\left(B_{j}^{\prime}\right)=$ $\left\{a_{j}^{\prime}, b_{j}^{\prime}, c_{j}^{\prime}\right\}$ with $a_{j}^{\prime} F_{i}^{\prime} b_{j}^{\prime} \subset B_{j}^{\prime}$. Then since $G$ is 4 -connected, $B_{j}^{\prime}-\left\{b_{j}^{\prime}, c_{j}^{\prime}\right\}$ is a chain 
of blocks with $a_{j}^{\prime}$ in an endblock not as a cutvertex. Hence by Lemma 6.2 we can find a 2 -walk $W_{j}^{\prime}$ of $B_{j}^{\prime}-\left\{b_{j}^{\prime}, c_{j}^{\prime}\right\}$ such that $a_{j}^{\prime}$ is only visited once. We attach $W_{j}^{\prime}$ to $T_{m}$ and we can similarly include vertices of those bridges of $M_{i}^{\prime}$ containing vertices of $F_{i}$.

It is now straightforward to verify that $T_{m}$ together with all those $W_{j}$ and $W_{j}^{\prime}$ forms a 2 -walk of $G$.

A corollary of this result is that every 4-connected graph in a surface of orientable or nonorientable genus $g$ with representativity at least $48\left(2^{g}-1\right)$ has a spanning tree of maximum degree 3 , which generalizes and improves a result of [4] (and so implies a conjecture of [14]).

Finally we deal with 3 -walks in 3-connected graphs. For convenience we view a $k$-walk as an eulerian graph by doubling or tripling an edge if it is used twice or three times. Ellingham (personal communication) asked if a 3-connected graph in a surface with large representativity has a 3 -walk? Here we answer this question in the affirmative.

Theorem 6.4. Let $G$ be a 3-connected graph embedded in a surface $\Sigma$ with eg $(G, \Sigma)$ $=g$ and $\rho(G, \Sigma) \geq 48\left(2^{g}-1\right)$. Then $G$ has a 3 -walk.

Sketch of a proof. By Theorem 4.3, $G$ contains a set of planarizing cycles $C_{1}, \cdots$, $C_{m}$ and cycles $D_{i}^{\prime 2}$ and $D_{i}^{2}$. Note that $d=5$. Define $H, H_{i}, M_{i}$ in the same way as in the proof of Theorem 5.1. Let $H^{\prime \prime}$ be the graph obtained from $H$ by deleting all $M_{i}$. Then each component of $H^{\prime \prime}$ is a 2-connected plane graph, and every 2-cut of $H^{\prime \prime}$ is contained in some $D_{i}^{\prime 2}$ or $D_{i}^{2}$. Let $M_{i}^{\prime \prime}$ be obtained from $M_{i}$ by deleting all $M_{j}$ which is contained in $M_{i}$. Then $M_{i}^{\prime \prime}$ is a 2-connected plane graph, and every 2-cut of $M_{i}^{\prime \prime}$ is contained in some $D_{j}^{\prime 2}$ or $D_{j}^{2}$. We now prove the following statement.

Let $K$ be a component of $H^{\prime \prime}$ and $x$ be a vertex in $K$. Then $K$ contains a 3 -walk using $x$ at most twice such that for each $D_{i}^{\prime 2} \subset K$ (respectively, each $D_{i}^{2} \subset K$ ) the following hold.

(i) if $D_{i}^{2}$ (respectively, $D_{i}^{\prime 2}$ ) contains no 2-cut of $K$, then every vertex of $D_{i}^{2}$ (respectively, $D_{i}^{\prime 2}$ ) is used at most twice, and

(ii) for every maximal 2-cut $\{u, v\} \subset D_{i}^{2}$ (respectively, $\{u, v\} \subset D_{i}^{\prime 2}$ ) and the $\{u, v\}$-bridge $B$ of $K$ not containing any other $D_{j}^{2}$ or $D_{j}^{\prime 2}$, the vertices in $B-\{u, v\}$ are used at most twice.

First, for every maximal 2-cut $\{u, v\}$ of $K$ contained in some $D_{i}^{2}$ or $D_{i}^{\prime 2}$, we replace with an edge $u v$ the $\{u, v\}$-bridge of $K$ not containing $D_{j}^{2}$ or $D_{j}^{\prime 2}$ for any $j \neq i$. This reduces $K$ to a 3 -connected plane graph which, by Lemma 6.1, contains a 2 -walk $W$.

Second, we extend $W$ to include the vertices in $W$-bridges of $K$ containing edges of $D_{i}^{2}$. Let $B_{1}, B_{2}, \cdots, B_{n}$ be the $W$-bridges of $K$ appearing on $D_{i}^{2}$ in this order. Let $a\left(B_{j}\right)=\left\{a_{j}, b_{j}\right\}$ with $a_{j} D_{i}^{2} b_{i} \subset B_{j}$. We may assume, by symmetry, that $x \notin\left\{a_{1}, \cdots, a_{n}\right\}$. Since $G$ is 3 -connected, $B_{j}-b_{j}$ is a chain of blocks with $a_{j}$ in an endblock not as a cutvertex.

If the edge $a_{j} b_{j}$ is not used by $W$, then by Lemma $6.2, B_{j}-b_{j}$ contains a 2 walk $W_{j}$ using $a_{j}$ only once. If the edge $a_{j} b_{j}$ is used by $W$, then $B_{j}+a_{j} b_{j}$ is a circuit graph, where $a_{j} D_{i}^{2} b_{j}+a_{j} b_{j}$ is the outercycle. Now by Lemma $6.1, B_{j}+a_{j} b_{j}$ contains a 2 -walk using $a_{j}$ and $b_{j}$ just once and using all edges of $a_{j} D_{i}^{2} b_{j}+a_{j} b_{j}$, and let $W_{j}$ be obtained from the 2 -walk by deleting the edge $a_{j} b_{j}$. 
Now the union of $W$ and all $W_{j}$ 's includes all vertices in $W$-bridges of $K$ containing edges of $D_{i}^{2}$. Similarly we can extend $W$ to include all vertices in $W$-bridges of $K$ containing an edge of $D_{i}^{\prime 2}$. Hence we get the desired 3-walk of $K$ as in the statement.

Since $G$ is 3 -connected, there is an edge from $x_{i} \in V\left(M_{i}\right)$ to $y_{i} \in D_{i}^{2}\left(\right.$ or $\left.y_{i} \in D_{i}^{\prime 2}\right)$ such that $y_{i}$ is used at most twice by 3 -walks in $M_{l}$ for $l<i$ (where $M_{0}=H^{\prime \prime}$ ). In a similar way, we can find 3-walks in $M_{i}^{\prime \prime}$ such that (i) and (ii) above hold with $x, D_{j}^{\prime 2}, D_{j}^{2}$ being replaced by $x_{i}, D_{j}^{\prime 2} \subset M_{i}^{\prime \prime}, D_{j}^{2} \subset M_{i}^{\prime \prime}$.

Now by combining the 3 -walks in $H^{\prime \prime}, M_{i}^{\prime \prime}$ together with the edges $x_{i} y_{i}$ we produce a 3 -walk in $G$.

We point out here that Theorem 6.4 also generalizes a result of Thomassen [14] on spanning trees in triangulations.

\section{ACKNOWLEDGMENT}

I am grateful to the referee for his valuable suggestions.

\section{REFERENCES}

1. J. A. Bondy and U. S. R. Murty, Graph Theory with Applications. American Elsevier, 1976. MR 54:117

2. R. Brunet, M. N. Ellingham, Z. Gao, A. Metzlar, and R. B. Richter, Spanning planar subgraphs of graphs in the torus and Klein bottle. J. Combin. Theory Ser. B 65 (1965) 7-22. MR 96g:05045

3. R. A. Duke, On the genus and connectivity of hamiltonian graphs. Discrete Math., Vol. 2, No. 3 (1972) 199-206. MR 47:3221

4. M. N. Ellingham and Z. Gao, Spanning trees in locally planar triangulations. J. Combinat. Theory Ser. B, 61 (1994) 178-198. MR 95m:05080

5. Z. Gao and R. B. Richter, 2-Walks in circuit graphs. J. Combin. Theory Ser. B 62 (1994) 259-267. MR 96e:05093

6. B. Grünbaum, Polytopes, graphs, and complexes. Bull. Amer. Math. Soc. 76 (1970) 11311201. MR 42:959

7. J. C. Molluzzo, Problem. in "Second International Conference on Combinatorial Mathematics" (A. Gewirtz and L. V. Quintas, Eds.) pp. 556-567, The New York Academy of Sciences, New York, 1979. MR 80j:05003

8. C. St. J. A. Nash-Williams, Unexplored and semi-explored territories in graph theory. New Directions in Graph Theory, Academic Press, New York (1973) 169-176. MR 52:7944

9. M. D. Plummer, Problem in infinite and finite sets. Coll. Math. Soc. J. Bolyai 10, Vol. III (A. Hajnal, R. Rado and V. T. Sós, Eds.), North-Holland, Amsterdam (1975) 1549-1550. MR 50:12526

10. R. Thomas and X. Yu, 4-Connected projective-planar graphs are hamiltonian. J. Combinat. Theory Ser. B 62 (1994) 114-132. MR 95j:05133

11. R. Thomas and X. Yu, 5-connected toroidal graphs are hamiltonian. J. Combinat. Theory Ser. B., in press.

12. C. Thomassen, A theorem on paths in planar graphs. J. Graph Theory 7 (1983) 169-176. MR 84i:05075

13. C. Thomassen, Embeddings of graphs with no short noncontractible cycles. J. Combinat. Theory Ser B 48 (1990) 155-177. MR 91b:05069

14. C. Thomassen, Trees in triangulations. J. Combinat. Theory Ser. B 60 (1994) 56-62. MR 95e:05039

15. C. Thomassen, 5-Coloring maps on surfaces. J. Combinat. Theory Ser. B 59 (1993) 89-105. MR 94h:05031 
16. W. T. Tutte, A theorem on planar graphs. Trans. Amer. Math. Soc. 82 (1956) 99-116. MR 18:408e

17. H. Whitney, A theorem on graphs. Ann. of Math. 32 (1931) 378-390.

School of Mathematics, Georgia Institute of Technology, Atlanta, Georgia 30332

Current address: Department of Mathematics, Vanderbilt University, Nashville, Tennessee 37240

E-mail address: yu@math.vanderbilt.edu 